\title{
Circle homeomorphisms and shears
}

\author{
DRAGOMIR ŠARIĆ
}

We give parameterizations of homeomorphisms, quasisymmetric maps and symmetric maps of the unit circle in terms of shear coordinates for the Farey tesselation.

\section{Introduction}

The space Homeo $\left(S^{1}\right)$ of orientation preserving homeomorphisms of the unit circle $S^{1}$ is a classical topological group which is of interest in various fields of mathematics; see Ghys [11]. Its subgroup QS $\left(S^{1}\right)$ of quasisymmetric maps of $S^{1}$ plays a fundamental role in the Teichmüller theory of Riemann surfaces; see Ahlfors [2], Bers [3] and Gardiner and Lakic [9]. In fact, the universal Teichmüller space consists of all quasisymmetric maps which fix three distinguished points on $S^{1}$ namely it is isomorphic to $\operatorname{Möb}\left(S^{1}\right) \backslash \mathrm{QS}\left(S^{1}\right)$, where $\operatorname{Möb}\left(S^{1}\right)$ is the group of (orientation preserving) Möbius maps which preserve $S^{1}$ [3]. The subgroup $\operatorname{Sym}\left(S^{1}\right)$ of symmetric maps plays a prominent role in studying Teichmüller spaces of real dynamical systems; see Gardiner and Sullivan [10], Earle, Gardiner and Lakic [6] and Gardiner and Jiang [8].

The main results in this article are explicit parametrizations of the three spaces $\operatorname{Möb}\left(S^{1}\right) \backslash \operatorname{Homeo}\left(S^{1}\right), \operatorname{Möb}\left(S^{1}\right) \backslash \mathrm{QS}\left(S^{1}\right)$ and $\operatorname{Möb}\left(S^{1}\right) \backslash \operatorname{Sym}\left(S^{1}\right)$ in terms of shear coordinates for the Farey tesselation of the hyperbolic plane $\mathbf{H}$. To our best knowledge these are the only known explicit parametrizations of the above spaces. The unit circle $S^{1}$ is the boundary at infinity of $\mathbf{H}$.

The shear of a pair $\left(\Delta, \Delta_{1}\right)$ of ideal hyperbolic triangles $\Delta$ and $\Delta_{1}$ in $\mathbf{H}$ with disjoint interiors and a common boundary side $e$ is the signed hyperbolic distance between the orthogonal projections of the third vertices of $\Delta$ and $\Delta_{1}$ onto $e$ (see Thurston [17], Bonahon [4], Penner [14] or Section 3). The Farey tesselation $\mathcal{F}$ is a locally finite ideal geodesic triangulation of $\mathbf{H}$ which is preserved by the hyperbolic reflections in geodesics of $\mathcal{F}$ (see, for example, Penner [15]). Note that the shear of each pair of adjacent complementary triangles of $\mathcal{F}$ is zero. The set of geodesics of $\mathcal{F}$ is naturally partitioned into Farey generations (see [15] or Section 3). 
A homeomorphism $h: S^{1} \rightarrow S^{1}$ induces a real-valued function $s_{h}: \mathcal{F} \rightarrow \mathbf{R}$, called shear function, whose domain is the Farey tesselation $\mathcal{F}$. Each geodesic $e \in \mathcal{F}$ is the common boundary side of a pair $\left(\Delta, \Delta_{1}\right)$ of complementary triangles of $\mathcal{F}$. We define $s_{h}(e)$ to be the shear of the image pair $\left(h(\Delta), h\left(\Delta_{1}\right)\right)$. Characterizing shear functions $s: \mathcal{F} \rightarrow \mathbf{R}$ that arise from homeomorphisms and characterizing shear functions that arise from quasisymmetric maps of $S^{1}$ were fairly known problems. We find both characterizations. (For a punctured surfaces $S^{\prime}$, the Teichmüller space $T\left(S^{\prime}\right)$ is parameterized using shears on locally finite tesselations by Thurston [17] and Penner [14]; in the case of a closed surfaces $S$, Thurston [16] and Bonahon [4] gave a parameterization of $T(S)$ using shears on locally infinite tesselations.)

A fan of geodesics in $\mathcal{F}$ with tip $p \in S^{1}$ consists of all geodesics of $\mathcal{F}$ which have one endpoint $p$. Each fan in $\mathcal{F}$ has a natural ordering as follows. Fix a horocycle $C$ with center at $p$ whose orientation is such that the corresponding horoball is to the left of $C$. If $e, e^{\prime}$ are two geodesics with common endpoint $p$, then we define $e<e^{\prime}$ if the point $e \cap C$ comes before the point $e^{\prime} \cap C$ on $C$, otherwise $e^{\prime}<e$. The natural ordering on a fan induces a bijective correspondence of the geodesics of the fan with the integers $\mathbf{Z}$, and any two such correspondences differ by a translation in $\mathbf{Z}$. For each fan in $\mathcal{F}$ we fix one such correspondence.

Theorem A A shear function $s: \mathcal{F} \rightarrow \mathbf{R}$ is induced by a quasisymmetric map of $S^{1}$ if and only if there exists $M \geq 1$ such that for each fan of geodesics $\left\{e_{n}\right\}_{n \in \mathbf{Z}}$ in $\mathcal{F}$ and for all $m, k \in \mathbf{Z}$, we have

$$
\frac{1}{M} \leq e^{s_{m}} \frac{1+e^{s_{m+1}}+\cdots+e^{s_{m+1}+\cdots+s_{m+k}}}{1+e^{-s_{m-1}}+\cdots+e^{-s_{m-1}-\cdots-s_{m-k}}} \leq M
$$

where $s_{n}=s\left(e_{n}\right)$.

Moreover, $s: \mathcal{F} \rightarrow \mathbf{R}$ is induced by a symmetric map of $S^{1}$ if and only if

$$
e^{s_{m}} \frac{1+e^{s_{m+1}}+\cdots+e^{s_{m+1}+\cdots+s_{m+k}}}{1+e^{-s_{m-1}}+\cdots+e^{-s_{m-1}-\cdots-s_{m-k}}} \rightrightarrows 1
$$

as the Farey generations of $e_{m-k}$ and $e_{m+k}$ go to infinity.

For a fan $\left\{e_{n}\right\}_{n \in \mathbf{Z}}$ in $\mathcal{F}$ with tip $p$, we define

$$
s(p ; m, k)=e^{s_{m}} \frac{1+e^{s_{m+1}}+\cdots+e^{s_{m+1}+\cdots+s_{m+k}}}{1+e^{-s_{m-1}}+\cdots+e^{-s_{m-1}-\cdots-s_{m-k}}}
$$

for $m, k \in \mathbf{Z}$. Let $C$ be a horoball with center at $h(p)$ where $h$ is a quasisymmetric map which induces $s$. If $k \geq 0$, then $s(p ; m, k)$ is the ratio of the length of the horocyclic arc on $C$ between $h\left(e_{m+k+1}\right) \cap C$ and $h\left(e_{m}\right) \cap C$ to the length of the horocyclic arc 
on $C$ between $h\left(e_{m-k-1}\right) \cap C$ and $h\left(e_{m}\right) \cap C$. If $k<0$, then $s(p ; m, k)$ is the ratio of the length of the horocyclic arc on $C$ between $h\left(e_{m+k-1}\right) \cap C$ and $h\left(e_{m}\right) \cap C$ to the length of the horocyclic arc on $C$ between $h\left(e_{m-k+1}\right) \cap C$ and $h\left(e_{m}\right) \cap C$. Define

$$
M_{S}(p)=\sup _{m, k \in \mathbf{Z}} s(p ; m, k) .
$$

If $M_{S}(p)<\infty$ then we say that $s$ satisfies $M_{S}(p)$-condition at the fan with tip $p$. Theorem A states that a shear map $s: \mathcal{F} \rightarrow \mathbf{R}$ induces a quasisymmetric map of $S^{1}$ if and only if

$$
M_{S}=\sup _{p} M_{S}(p)<\infty
$$

where the supremum is over all fans of $\mathcal{F}$. The $M_{s}(p)$-condition is localized in a fan of geodesics with tip $p$ and the only additional information is that single $M_{s}=$ $\sup _{p} M_{s}(p)$ works for all fans simultaneously. In particular, the characterization of quasisymmetry does not require any information about the relationship between shears on geodesics of $\mathcal{F}$ not belonging to the same fan.

We now give an interpretation of the Teichmüller topology of the universal Teichmüller space $\operatorname{Möb}\left(S^{1}\right) \backslash \mathrm{QS}\left(S^{1}\right)$ within the framework of Theorem A. Theorem A parametrizes $\operatorname{Möb}\left(S^{1}\right) \backslash \mathrm{QS}\left(S^{1}\right)$ by the space $\mathcal{X}$ of all shear functions $s: \mathcal{F} \rightarrow \mathbf{R}$ which satisfy (1). We use the quantity $s(p ; m, k)$ to introduce a topology on $\mathcal{X}$ such that the parametrization of $\operatorname{Möb}\left(S^{1}\right) \backslash \mathrm{QS}\left(S^{1}\right)$ by $\mathcal{X}$ is a homeomorphism. For $s_{1}, s_{2} \in \mathcal{X}$ define

$$
M_{s_{1}, s_{2}}(p)=\sup _{m, k \in \mathbf{Z}}\left(\max \left\{\frac{s_{1}(p ; m, k)}{s_{2}(p ; m, k)}, \frac{s_{2}(p ; m, k)}{s_{1}(p ; m, k)}\right\}\right) .
$$

Theorem B Let $h_{n}, h \in \operatorname{Möb}\left(S^{1}\right) \backslash \mathrm{QS}\left(S^{1}\right)$. Then $h_{n} \rightarrow h$ as $n \rightarrow \infty$ in the Teichmüller topology if and only if $M_{s, s_{n}}=\sup _{p} M_{s, s_{n}}(p) \rightarrow 1$ as $n \rightarrow \infty$.

Remark Kahn and Markovic [12] estimated, in terms of shears, the Teichmüller and Weil-Petersson distances between two finite punctured Riemann surfaces, and their estimate is independent of the topological complexity of the surfaces. Theorem B describes the Teichmüller topology on the universal Teichmüller space in terms of shears.

Characterization of homeomorphisms involves more information than the parametrization of quasisymmetric homeomorphisms given by Theorem A. A chain of geodesics in $\mathcal{F}$ is a sequence $\left\{e_{n}\right\}_{n \in \mathbf{N}}$ of distinct, adjacent geodesics of $\mathcal{F}$. 
Theorem $\mathbf{C}$ A shear function $s: \mathcal{F} \rightarrow \mathbf{R}$ is induced by a homeomorphism of $S^{1}$ if and only if for each chain $\left\{e_{n}\right\}_{n \in \mathbf{N}}$ in $\mathcal{F}$ we have

$$
\sum_{n=1}^{\infty} e^{s_{1}^{n}+s_{2}^{n}+\cdots+s_{n}^{n}}=\infty
$$

where $s_{i}^{n}= \pm s\left(e_{i}\right)$. More precisely if $e_{n}<e_{n+1}$ then $s_{n}^{n}=s\left(e_{n}\right)$; otherwise $s_{n}^{n}=$ $-s\left(e_{n}\right)$. For $n>1$ and $i<n, s_{i}^{n}=s\left(e_{i}\right)$ if either $e_{i}<e_{i+1}$ and the number of times we change fans from $e_{i}$ to $e_{n+1}$ is even, or $e_{i}>e_{i+1}$ and the number of times we change fans is odd; otherwise $s_{i}^{n}=-s\left(e_{i}\right)$.

A locally finite ideal triangulation of $\mathbf{H}$ with a distinguished oriented geodesic (edge) is called a tesselation. The space of all tesselations is isomorphic to the space $\operatorname{Homeo}\left(S^{1}\right)$ by assigning to a tesselation $\tau$ a homeomorphism of $S^{1}$ (called the characteristic map) which maps the Farey tesselation $\mathcal{F}$ to the tesselation $\tau$ of $\mathbf{H}$ such that the distinguished oriented geodesic of $\mathcal{F}$ is mapped onto the distinguished oriented geodesic of $\tau$ (see Penner [15]). A decorated tesselation is a tesselation together with an arbitrary assignment of a horocycle at each vertex of the tesselation (see [15]).

Let $C_{1}$ and $C_{2}$ be two horocycles with different centers and let $g$ be the geodesic whose endpoints are at the centers of $C_{1}$ and $C_{2}$. Then the lambda length $\lambda(g)$ of $g$ is defined by

$$
\lambda(g)=e^{-2 \delta\left(C_{1}, C_{2}\right)}
$$

where $\delta\left(C_{1}, C_{2}\right)$ is the signed hyperbolic distance between $G_{1}=g \cap C_{1}$ and $G_{2}=$ $g \cap C_{2}$. The sign of $\delta\left(C_{1}, C_{2}\right)$ is positive if the geodesic arc between $G_{1}$ and $G_{2}$ is outside $C_{1}$, otherwise the sign is negative. Two geodesics $g$ and $g_{1}$ form a wedge in $\mathbf{H}$ if they have a common endpoint. Let $C$ be a horocycle with center at the common endpoint of the wedge formed by $g$ and $g_{1}$. The horocyclic length $\alpha\left(g, g_{1}\right)$ of the wedge $g, g_{1}$ is the length of the arc of $C$ between $g$ and $g_{1}$. A decorated tesselation $\tilde{\tau}$ determines an assignment of lambda lengths to the geodesics of the underlining tesselation $\tau$ and an assignment of horocyclic lengths to the wedges of $\tau$. This in turn defines an assignment of lambda lengths to the geodesics of the Farey tesselation $\mathcal{F}$ by the pullback with the characteristic map as well as the assignment of horocyclic lengths to the wedges of $\mathcal{F}$ (see Penner [15; 14]).

Two decorated tesselations $\tilde{\tau}_{1}$ and $\tilde{\tau}_{2}$ induce the same lambda lengths on $\mathcal{F}$ if and only if $\tilde{\tau}_{1}$ is the image under an element of $\operatorname{Möb}\left(S^{1}\right)$ of $\tilde{\tau}_{2}$. It is clear that not every assignment of lambda lengths on the Farey tesselation $\mathcal{F}$ will give a decorated tesselation such that the characteristic map extends to a homeomorphism of $S^{1}$. In fact the underlying tesselation is not in general an ideal triangulation of $\mathbf{H}$. Penner [15] posed 
the problem of determining which lambda lengths will give characteristic maps that are homeomorphisms or quasisymmetric maps of $S^{1}$. Penner and Sullivan [15, Theorem 6.4] showed that if lambda lengths are "pinched", namely if there is $K \geq 1$ such that $1 / K \leq \lambda(e) \leq K$ for all $e \in \mathcal{F}$, then the characteristic map is quasisymmetric. We find necessary and sufficient conditions on the lambda lengths such that the characteristic maps are homeomorphisms, quasisymmetric or symmetric maps of $S^{1}$.

Theorem D A lambda length function $\lambda: \mathcal{F} \rightarrow \mathbf{R}^{+}$induces a homeomorphism of $S^{1}$ if and only if for each chain of geodesics $\left\{e_{n}\right\}_{n \in \mathbf{N}}$ in $\mathcal{F}$ we have

$$
\sum_{n=1}^{\infty}\left(\lambda_{n}^{-1 / 2} \lambda_{n-1}^{1 / 2} \cdots \lambda_{1}^{(-1)^{n} / 2}\right) \alpha_{n}=\infty
$$

where $\lambda_{i}=\lambda\left(e_{i}\right)$ and $\alpha_{n}$ is the horocyclic length of the wedge bounded by $e_{n}$ and $e_{n+1}$.

In the above theorem we used horocyclic length $\alpha_{n}$ together with the lambda lengths. We note that horocyclic lengths are expressed as rational functions of lambda lengths (see Penner [14; 15, Section 6]). Indeed, if $g_{1}, g_{2}, g_{3}$ are boundary geodesics of an ideal hyperbolic triangle with decorations then, from [14], we have

$$
\alpha\left(g_{1}, g_{2}\right)=\frac{2 \lambda\left(g_{3}\right)}{\lambda\left(g_{1}\right) \lambda\left(g_{2}\right)} .
$$

Thus the series in the above theorem is completely determined in terms of lambda lengths.

The following theorem gives necessary and sufficient conditions on horocyclic lengths such that the characteristic maps are quasisymmetric and symmetric. We note that it possible to express the same condition in terms of lambda lengths using the formula above.

Theorem $\mathbf{E}$ A lambda length function $\lambda: \mathcal{F} \rightarrow \mathbf{R}$ induces a quasi-symmetric map of $S^{1}$ if and only if there exists $K \geq 1$ such that for each fan of geodesics $\left\{e_{n}\right\}_{n \in \mathbf{Z}}$ in $\mathcal{F}$ and for all $m \in \mathbf{Z}$ and $k \in \mathbf{N}$ we have

$$
\frac{1}{K} \leq \frac{\alpha\left(e_{m}, e_{m+1}\right)+\alpha\left(e_{m+1}, e_{m+2}\right)+\cdots+\alpha\left(e_{m+k}, e_{m+k+1}\right)}{\alpha\left(e_{m}, e_{m-1}\right)+\alpha\left(e_{m-1}, e_{m-2}\right)+\cdots+\alpha\left(e_{m-k}, e_{m-k-1}\right)} \leq K .
$$

Moreover, $\lambda: \mathcal{F} \rightarrow \mathbf{R}$ induces a symmetric map of $S^{1}$ if and only if

$$
\frac{\alpha\left(e_{m}, e_{m+1}\right)+\alpha\left(e_{m+1}, e_{m+2}\right)+\cdots+\alpha\left(e_{m+k}, e_{m+k+1}\right)}{\alpha\left(e_{m}, e_{m-1}\right)+\alpha\left(e_{m-1}, e_{m-2}\right)+\cdots+\alpha\left(e_{m-k}, e_{m-k-1}\right)} \rightarrow 1
$$

as the Farey generations of $e_{m+k}$ and $e_{m-k}$ go to infinity independently of the fan. 
Acknowledgements This work was supported in part by PSC CUNY grant PSCREG40-136.

\section{Quasisymmetric maps and barycentric extension}

In the rest of the paper the hyperbolic plane is identified with the upper half-plane model $\mathbf{H}:=\{z=x+i y \mid y>0\}$ endowed with the metric $\rho(z)=|d z| / y$. The boundary at infinity $\partial_{\infty} \mathbf{H}=\widehat{\mathbf{R}}=\mathbf{R} \cup\{\infty\}$ is naturally identified with the unit circle $S^{1}$. Any two identifications of $\widehat{\mathbf{R}}$ and $S^{1}$ differ by the postcomposition by a Möbius map of $S^{1}$. We choose 0,1 and $\infty$ to be the three distinguished points on $\hat{\mathbf{R}}$.

Let $h: \hat{\mathbf{R}} \rightarrow \widehat{\mathbf{R}}$ be a homeomorphism that fixes $\infty$ and let $M \geq 1$. Then $h: \hat{\mathbf{R}} \rightarrow \widehat{\mathbf{R}}$ is said to be $M$-quasisymmetric if

$$
\frac{1}{M} \leq \frac{h(x+t)-h(x)}{h(x)-h(x-t)} \leq M
$$

for all $x \in \mathbf{R}$ and $t>0$ (see Ahlfors [2]).

The universal Teichmüller space $T(\mathbf{H})$ is the set of all quasisymmetric maps of $\widehat{\mathbf{R}}$ that fix 0,1 and $\infty$. A sequence $h_{n} \in T(\mathbf{H})$ converges to the basepoint id $\in T(\mathbf{H})$ in the Teichmüller topology if $h_{n}$ are $M_{n}$-quasisymmetric with $M_{n} \rightarrow 1$ as $n \rightarrow \infty$. A sequence $h_{n} \in T(\mathbf{H})$ converges to $h \in T(\mathbf{H})$ in the Teichmüller topology if $h_{n} \circ h^{-1} \rightarrow \mathrm{id}$ as $n \rightarrow \infty$ in the above sense.

A quasisymmetric map $h: \widehat{\mathbf{R}} \rightarrow \widehat{\mathbf{R}}$ extends to a quasiconformal map $f: \mathbf{H} \rightarrow \mathbf{H}$, and conversely a quasiconformal map $f: \mathbf{H} \rightarrow \mathbf{H}$ extends by continuity to a quasisymmetric map $h: \widehat{\mathbf{R}} \rightarrow \widehat{\mathbf{R}}$ (see [2]). The extension of $h: \widehat{\mathbf{R}} \rightarrow \widehat{\mathbf{R}}$ to a quasiconformal map of $\mathbf{H}$ is not unique. Douady and Earle [5] defined a particularly nice extension operator from quasisymmetric maps of $\widehat{\mathbf{R}}$ into quasiconformal maps of $\mathbf{H}$ called the barycentric extension.

For a homeomorphism $h: \hat{\mathbf{R}} \rightarrow \widehat{\mathbf{R}}$, denote by $\operatorname{ex}(h): \mathbf{H} \rightarrow \mathbf{H}$ its barycentric extension introduced in [5]. We recall several properties of ex $(h)$ that are obtained by Douady and Earle [5]. The barycentric extension $\operatorname{ex}(h)$ is a real-analytic diffeomorphism of $\mathbf{H}$ which is quasiconformal if and only if $h$ is quasisymmetric. Moreover, the extension is conformally natural in the sense that $\operatorname{ex}(A \circ h \circ B)=A \circ \operatorname{ex}(h) \circ B$ for all $A, B \in \mathrm{PSL}_{2}(\mathbf{R})$ and for all homeomorphisms $h: \hat{\mathbf{R}} \rightarrow \widehat{\mathbf{R}}$. In addition, if $h_{n} \rightarrow h$ as $n \rightarrow \infty$ pointwise on $\widehat{\mathbf{R}}$ then $\operatorname{ex}\left(h_{n}\right) \rightarrow \operatorname{ex}(h)$ as $n \rightarrow \infty$ in the $C^{\infty}$-topology on $C^{\infty}$ maps of $\mathbf{H}$. In particular, Beltrami coefficients $\mu\left(\operatorname{ex}\left(h_{n}\right)\right)$ of ex $\left(h_{n}\right)$ converge uniformly on compact subsets of $\mathbf{H}$ to the Beltrami coefficient $\mu(\operatorname{ex}(h))$ of ex $(h)$. 
Remark 2.1 For our purposes the barycentric extension serves quite well. Kahn and Markovic [12] constructed another quasiconformal extension in the case when the quasisymmetric maps are invariant under cofinite Fuchsian group in order to be able to estimate the norm of the corresponding Beltrami coefficient.

The following lemma is obtained by Markovic [13] (see also Douady and Earle [5] and Abikoff, Earle and Mitra [1]).

Lemma 2.2 Let $h_{n}: \widehat{\mathbf{R}} \rightarrow \widehat{\mathbf{R}}$ be a sequence of homeomorphisms which fix 0,1 and $\infty$, and let $\mu_{n}$ be Beltrami coefficients of the barycentric extensions $\operatorname{ex}\left(h_{n}\right)$ of $h_{n}$. If there exists $c_{0} \geq 1$ such that

$$
--c_{0} \leq h_{n}(-1) \leq-\frac{1}{c_{0}}
$$

then there exists a neighborhood $U$ of the imaginary unit $i \in \mathbf{H}$ and a constant $0<c<1$ such that

$$
\left\|\left.\mu_{n}\right|_{U}\right\|_{\infty} \leq c<1
$$

for all $n$.

Proof We note that the angle distance with respect to $i \in \mathbf{H}$ between all pairs of consecutive points in $\{\infty,-1,0,1\} \subset \widehat{\mathbf{R}}$ is bounded below by a constant less than $\pi$ and bounded above by $\pi$. Then [13, Lemma 3.6] directly implies the desired conclusion.

\section{Farey tesselation and the shear map}

Let $\Delta_{0}$ be ideal geodesic triangle in $\mathbf{H}$ with vertices 0,1 and $\infty$. Let $\Gamma$ be the group generated by hyperbolic reflections in the sides of $\Delta_{0}$. The Farey tesselation $\mathcal{F}$ is an ideal triangulation of $\mathbf{H}$ which is the $\Gamma$-orbit of the boundary sides of $\Delta_{0}$. In other words, each geodesic in $\mathcal{F}$ is obtained by applying finitely many inversions in boundary geodesics of $\Delta_{0}$ to a boundary geodesic of $\Delta_{0}$ (see, for example, [15]). The set of endpoints of the geodesics in $\mathcal{F}$ (vertices of $\mathcal{F}$ ) is $\widehat{\mathbf{Q}}=\mathbf{Q} \cup\{\infty\}$.

We define Farey generation of geodesics in $\mathcal{F}$ as follows. A boundary geodesic of $\Delta_{0}$ has Farey generation 0 . If a geodesic of $\mathcal{F}$ is obtained by $n$ reflections of a geodesic of generation 0 (where $n$ is the smallest such number) then its Farey generation is $n$.

Let $\left(\Delta_{1}, \Delta_{2}\right)$ be a pair of ideal geodesic triangles in $\mathbf{H}$ with disjoint interiors and a common boundary side. Let $A \in \operatorname{PSL}_{2}(\mathbf{R})$ be the unique Möbius map that sends $\Delta_{1}$ onto the triangle with vertices $-1,0$ and $\infty$, and that sends the common boundary side of $\left(\Delta_{1}, \Delta_{2}\right)$ onto the geodesic with vertices 0 and $\infty$. Then $A\left(\Delta_{2}\right)$ has vertices 
$0, e^{r}$ and $\infty$ for some $r \in \mathbf{R}$. The shear of the pair of triangles $\left(\Delta_{1}, \Delta_{2}\right)$ is by definition equal to $r$. Alternatively, the shear of a pair $\left(\Delta_{1}, \Delta_{2}\right)$ of adjacent triangles is the signed distance of the projections onto common boundary side $e$ of vertices of $\Delta_{1}$ and $\Delta_{2}$ opposite $e$, where $e$ is oriented to the left as seen from $\Delta_{1}$. Note that the shear of $\left(\Delta_{1}, \Delta_{2}\right)$ is equal to the shear of $\left(\Delta_{2}, \Delta_{1}\right)$. For example, any two adjacent triangles in the complement of the Farey tesselation $\mathcal{F}$ have shear 0 .

Let $h: \hat{\mathbf{R}} \rightarrow \hat{\mathbf{R}}$ be a homeomorphism. Every geodesic of $\mathbf{H}$ has exactly two distinct ideal endpoints on $\widehat{\mathbf{R}}$ and, conversely every two points on $\widehat{\mathbf{R}}$ determine a geodesic in $\mathbf{H}$. Thus, the space $\mathcal{G}$ of (oriented) geodesics in $\mathbf{H}$ is identified with the set of pairs of distinct points in $\widehat{\mathbf{R}}$. Therefore, the homeomorphism $h: \hat{\mathbf{R}} \rightarrow \widehat{\mathbf{R}}$ extends to a homeomorphism $h: \mathcal{G} \rightarrow \mathcal{G}$ of the space of geodesics $\mathcal{G}$. In particular, $h(\mathcal{F})$ is an ideal triangulation of $\mathbf{H}$ whose complementary triangles are $h\left(\Gamma\left(\Delta_{0}\right)\right)$.

Definition 3.1 Let $h: \hat{\mathbf{R}} \rightarrow \widehat{\mathbf{R}}$ be a homeomorphism. A geodesic $e \in \mathcal{F}$ is on the boundary of exactly two complementary triangles $\Delta_{1}, \Delta_{2}$. Then we assign to $e \in \mathcal{F}$ the shear of the pair $\left(h\left(\Delta_{1}\right), h\left(\Delta_{2}\right)\right)$ of triangles in $h\left(\Gamma\left(\Delta_{0}\right)\right)$. This determines a function

$$
s_{h}: \mathcal{F} \rightarrow \mathbf{R}
$$

which is called the shear function of $h$.

If we are given a shear between two adjacent triangles and the position of one of the triangles, the other triangle is uniquely determined. More generally, a pair of adjacent triangles with an assigned shear is determined up to a Möbius map because any ideal hyperbolic triangle can be mapped onto any other ideal hyperbolic triangle by a Möbius map.

If $h: \hat{\mathbf{R}} \rightarrow \widehat{\mathbf{R}}$ fixes 0,1 and $\infty$, then it is uniquely determined by the shear function $s_{h}: \mathcal{F} \rightarrow \mathbf{R}$. Given a shear function $s: \mathcal{F} \rightarrow \mathbf{R}$ there exists a unique injective (developing) map $h_{s}$ from the vertices $\widehat{\mathbf{Q}} \subset \widehat{\mathbf{R}}$ of the Farey tesselation $\mathcal{F}$ into $\widehat{\mathbf{R}}$ such that $h_{s}$ fixes 0,1 and $\infty$. The map $h_{s}$ realizes the shear function $s$ and it is called characteristic map of $s$ (see [15] or the next section for its definition).

\section{Homeomorphisms and shears}

We characterize shear functions $s: \mathcal{F} \rightarrow \mathbf{R}$ whose characteristic maps continuously extend to homeomorphisms of $\widehat{\mathbf{R}}$. An arbitrary function $s: \mathcal{F} \rightarrow \mathbf{R}$ induces a piecewise Möbius cocycle map $H_{s}: \mathbf{H} \rightarrow \mathbf{H}$ as follows. Recall that $\Delta_{0}$ is the ideal geodesic triangle with vertices 0,1 and $\infty$, and define $\left.H_{S}\right|_{\Delta_{0}}=$ id. Consider any other complementary triangle $\Delta \in \Gamma\left(\Delta_{0}\right)$ of $\mathcal{F}$. Let $l$ be the geodesic arc connecting the center of 
$\Delta_{0}$ to the center of $\Delta$. Let $\left\{e_{1}, e_{2}, \ldots, e_{n}\right\}$ be geodesics of $\mathcal{F}$ which intersect $l$ in the given order such that $e_{1}$ is a boundary side of $\Delta_{0}$ and $e_{n}$ is a boundary side of $\Delta$. We orient $e_{i}$ to the left as seen from $\Delta_{0}$. Then we set $\left.H_{s}\right|_{\Delta}=T_{e_{1}}^{s\left(e_{1}\right)} \circ T_{e_{2}}^{s\left(e_{2}\right)} \circ \cdots \circ T_{e_{n}}^{s\left(e_{n}\right)}$, where $T_{e_{i}}^{s\left(e_{i}\right)}$ is the hyperbolic translation with the oriented axis $e_{i}$ and the signed translation length $s\left(e_{i}\right)$. The map $H_{s}$ is not well-defined on the edges $\mathcal{F}$ since each edge $e$ is on the boundary of exactly two complementary triangles $\Delta_{e}^{1}$ and $\Delta_{e}^{2}$. We choose $\left.H_{S}\right|_{e}$ to be either $\left.H_{S}\right|_{\Delta_{e}^{1}}$ or $\left.H_{S}\right|_{\Delta_{e}^{2}}$. The cocycle map $H_{S}$ preserves separation properties of the triples of geodesics in $\mathcal{F}$. Therefore, $H_{s}$ extends to a monotone map $h_{s}: \widehat{\mathbf{Q}} \rightarrow \widehat{\mathbf{R}}$ which is called characteristic map of $s: \mathcal{F} \rightarrow \mathbf{R}$ (see Penner [15]).

Proposition 4.1 With the above notation, the characteristic map $h_{s}: \hat{\mathbf{Q}} \rightarrow \widehat{\mathbf{R}}$ extends by continuity to a homeomorphism of $\widehat{\mathbf{R}}$ if and only if $H_{s}: \mathbf{H} \rightarrow \mathbf{H}$ is surjective.

Proof Since $h_{s}: \hat{\mathbf{Q}} \rightarrow \widehat{\mathbf{R}}$ is order preserving on a dense subset $\hat{\mathbf{Q}}$ of $\widehat{\mathbf{R}} \equiv S^{1}$, it follows that if $h_{s}$ can be extended to a continuous map on $\hat{\mathbf{R}}$ then the extension is a homeomorphism.

If $H_{S}: \mathbf{H} \rightarrow \mathbf{H}$ is not onto, then there exists a maximal half-plane $P$ not contained in $H_{S}(\mathbf{H})$. It follows that the image $h_{s}(\widehat{\mathbf{Q}})$ does not intersect the interior of the interval on $\widehat{\mathbf{R}}$ which is the boundary at infinity of $P$. Therefore, the map $h_{s}: \hat{\mathbf{Q}} \rightarrow \widehat{\mathbf{R}}$ cannot be extended to a homeomorphism of $\widehat{\mathbf{R}}$.

Assume that $H_{s}: \mathbf{H} \rightarrow \mathbf{H}$ is onto. Let $x \in \hat{\mathbf{R}} \backslash \widehat{\mathbf{Q}}$. We need to show that $h_{s}$ extends to $x$. Let $P_{i}$ be a decreasing sequence of half-planes with boundary sides $e_{i} \in \mathcal{F}$ that accumulate at $x$, namely $\bigcap_{i} \overline{P_{i}}=x$. Since $H_{s}$ is order preserving on triples of geodesics in $\mathcal{F}$, it follows that $H_{S}\left(P_{i}\right)$ is a decreasing sequence of half-planes. If $\bigcap_{i} H_{s}\left(P_{i}\right) \neq \varnothing$ then $H_{s}(\mathbf{H}) \neq \mathbf{H}$, namely $H_{s}(\mathbf{H}) \cap\left(\bigcap_{i} H_{s}\left(P_{i}\right)\right)=\varnothing$. Thus $\bigcap_{i} H_{s}\left(P_{i}\right)=\varnothing$ and $\bigcap_{i} \overline{H_{s}\left(P_{i}\right)}$ is a single point $y \in \hat{\mathbf{R}}$. Then $h_{s}$ extends to $x$ by continuity such that $h_{s}(x)=y$.

Proof of Theorem $\mathbf{C}$ Using the above proposition we determine which shear maps induce homeomorphisms of $\widehat{\mathbf{R}}$. Assume that $H_{s}: \mathbf{H} \rightarrow \mathbf{H}$ is not onto. Then there exists a maximal half-plane $P$ of $\mathbf{H}$ which is not in the image of $H_{S}$. Let $l$ be the boundary geodesic of the half-plane $P$. Then there exists a chain $\left\{e_{n}\right\}_{n \in \mathbf{N}}$ of geodesics in $\mathcal{F}$ such that $H_{s}\left(e_{n}\right) \rightarrow l$ as $n \rightarrow \infty$. There are two possibilities for the sequence $e_{n}$. Either all $e_{n}$ 's share a common endpoint $x \in \hat{\mathbf{Q}} \subset \widehat{\mathbf{R}}$ for $n \geq n_{0}$ namely the subchain $e_{n}$, for $n \geq n_{0}$, is a part of a single fan, or $e_{n}$ 's accumulate to a point $x \in \hat{\mathbf{R}} \backslash \hat{\mathbf{Q}}$ (which is equivalent to saying that no infinite subsequence of $e_{n}$ 's shares a common endpoint ie no tail of $e_{n}$ 's is a part of a single fan). In both cases the existence of the half-plane $P$ is equivalent to the statement that $h_{s}$ does not extend to a continuous map at $x \in \hat{\mathbf{R}}$. 
Assume that we are in the first case. By precomposition with an element of $\mathrm{PSL}_{2}(\mathbf{Z})$, we can assume that $x=\infty$. In addition, we can assume that $H_{S}$ fixes 0,1 and $\infty$ by postcomposing with an element of $\mathrm{PSL}_{2}(\mathbf{R})$. Then $l$ has one endpoint $x=\infty$ and the other endpoint $\bar{y} \in \mathbf{R}$ with either $\bar{y}>1$ or $\bar{y}<0$. If $\bar{y}>1$, then

$$
\bar{y}=1+\sum_{n=1}^{\infty} e^{s\left(e_{1}\right)+\cdots+s\left(e_{n}\right)},
$$

where $e_{i} \in \mathcal{F}$ is a geodesic with endpoints $i$ and $\infty$ for $i \in \mathbf{N}$. If $\bar{y}<0$ then

$$
\bar{y}=-\sum_{n=0}^{-\infty} e^{-s\left(e_{0}\right)-\cdots-s\left(e_{n}\right)},
$$

where $e_{i} \in \mathcal{F}$ is a geodesic with endpoints $i$ and $\infty$ for $i \in \mathbf{Z}^{-} \cup\{0\}$. Since $e_{i}$ 's belong to a single fan, the number of times we change fans from $e_{i}$ to $e_{n+1}$ is zero. Thus $s_{i}^{n}=s\left(e_{i}\right)$ for $i>0$ and $s_{i}^{n}=-s\left(e_{i}\right)$ for $i \leq 0$. Therefore, $h_{s}$ is continuous at $x \in \widehat{\mathbf{R}}$ if and only if the series in (2) and the series in (3) diverge.

Assume now that we are in the second case. Namely, the chain $\left\{e_{n}\right\}_{n \in \mathbf{N}}$ does not have a subsequence which shares a common endpoint and $e_{n}$ 's accumulate at $x \in \hat{\mathbf{R}} \backslash \hat{\mathbf{Q}}$. In other words, no tail of $e_{n}$ 's is in a single fan. The part of $\mathbf{H}$ bounded by $e_{n}$ and $e_{n+1}$ is called a hyperbolic wedge.

Given a hyperbolic wedge, there is a unique foliation of the wedge by horocyclic arcs which lie on horocycles with centers at the common endpoint of the two boundary geodesics of the wedge. Consider the wedges whose boundaries are the adjacent geodesics in the chain $h_{s}\left(e_{n}\right)$ and foliate each wedge by horocyclic arcs as above. Fix a point $P_{1} \in h_{s}\left(e_{1}\right)$ and denote by $l\left(P_{1}\right)$ the leaf of the horocyclic foliation of the union of wedges that starts at $P_{1}$. Let $W_{n}$ be the hyperbolic wedge bounded by $h_{s}\left(e_{n}\right)$ and $h_{s}\left(e_{n+1}\right)$. We choose $P_{1}$ such that the length of $l\left(P_{1}\right) \cap W_{1}$ is $e^{s_{1}^{1}}$, where $s_{1}^{1}=s\left(e_{1}\right)$ if $e_{1}<e_{2}$, otherwise $s_{1}^{1}=-s\left(e_{1}\right)$ (see Figure 1 ).

Proposition 4.2 Under the above notation, the map $h_{s}$ continuously extends to $x \in$ $\widehat{\mathbf{R}} \backslash \widehat{\mathbf{Q}}$ if and only if the leaf $l\left(P_{1}\right)$ is of infinite length.

Proof Note that $h_{s}$ extends by continuity to $x \in \widehat{\mathbf{R}}$ if and only if $h_{s}\left(e_{n}\right)$ do not accumulate in $\mathbf{H}$.

Assume that $h_{s}$ extends continuously to $x \in \hat{\mathbf{R}}$. Then $h_{s}\left(e_{n}\right)$ do not accumulate in $\mathbf{H}$. Therefore, the arc $l\left(P_{1}\right)$ accumulates at $\partial \mathbf{H}$ and it is necessarily of infinite length. 


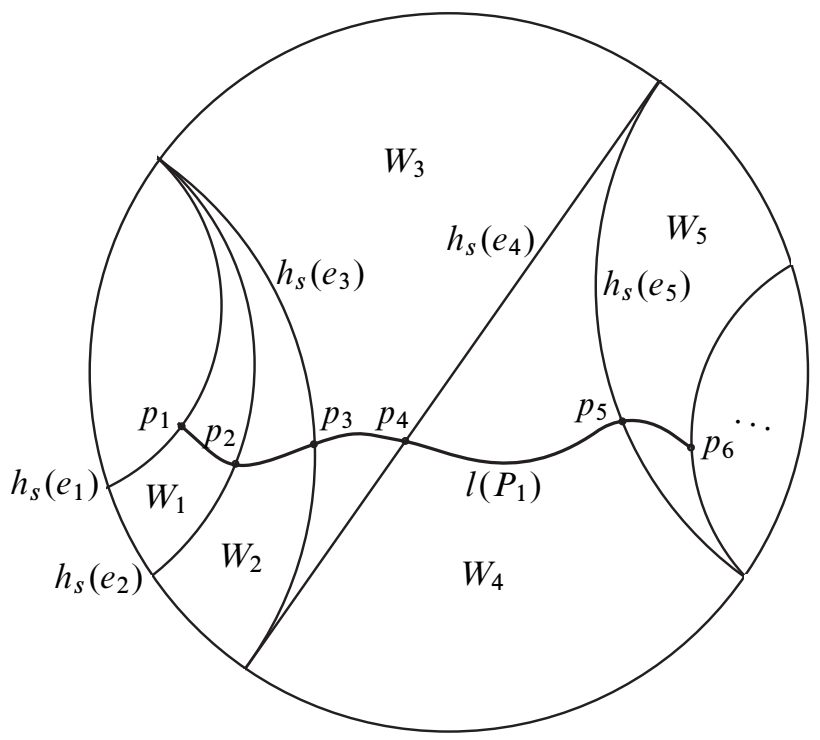

Figure 1: The leaf $l\left(P_{1}\right)$ of the foliation of $\bigcup_{n} W_{n}$ by horocyclic arcs

It remains to show that if $l\left(P_{1}\right)$ is of infinite length then $h_{s}$ extends to $x \in \widehat{\mathbf{R}}$ by continuity. Assume on the contrary that $h_{s}$ does not extend to $x \in \widehat{\mathbf{R}}$. This implies that $h_{s}\left(e_{n}\right)$ accumulate at a geodesic $g \subset \mathbf{H}$. We need to show that $l\left(P_{1}\right)$ has finite length.

Let $a$ be the geodesic arc which connects $h_{s}\left(e_{1}\right)$ with $g$ and that is orthogonal to both $h_{s}\left(e_{1}\right)$ and $g$. All the geodesics of the sequence $h_{s}\left(e_{n}\right)$ for $n \geq 2$ lie between $h_{s}\left(e_{1}\right)$ and $g$, and they intersect $a$. The angle of the intersection between $h_{s}\left(e_{n}\right)$ and $a$ is necessarily bounded away from 0 . We show that the length of $l\left(P_{1}\right)$ is comparable to the length of $a$ which finishes the proof.

Consider a hyperbolic wedge $W_{n}$ bounded with $h_{s}\left(e_{n}\right)$ and $h_{s}\left(e_{n+1}\right)$. Let $a_{n}=a \cap W_{n}$, and let $P_{n}^{\prime}=a \cap h_{s}\left(e_{n}\right)$. Then $P_{n}^{\prime}$ and $P_{n+1}^{\prime}$ are the endpoints of $a_{n}$. Let $P_{n}=$ $l\left(P_{1}\right) \cap h_{s}\left(e_{n}\right)$ and let $P_{n}^{\prime \prime}$ be the endpoint of the horocyclic arc in the wedge $W_{n}$ whose initial point is $P_{n}^{\prime}$ (see Figure 2). Let $d_{n}$ be the geodesic arc with endpoints $P_{n}$ and $P_{n}^{\prime}$, and let $d_{n}^{\prime}$ be the geodesic arc with endpoints $P_{n}^{\prime}$ and $P_{n}^{\prime \prime}$. Consider the hyperbolic triangle with vertices $P_{n}^{\prime}, P_{n+1}^{\prime}$ and $P_{n}^{\prime \prime}$. Since the angle at $P_{n}^{\prime \prime}$ is bounded away from 0 (by the uniform bound on the length of each $a_{n}$ ), it follows from the hyperbolic sine formula that there exists $C>0$ such that $\left|d_{n}^{\prime}\right| \leq C \cdot\left|a_{n}\right|$, where $\left|d_{n}^{\prime}\right|,\left|a_{n}\right|$ are the lengths of $d_{n}^{\prime}, a_{n}$, respectively. In addition, $\left|d_{n+1}\right|=\left|d_{n}\right|+\left|P_{n}^{\prime \prime} P_{n+1}^{\prime}\right| \leq\left|d_{n}\right|+\left|d_{n}^{\prime}\right|+\left|a_{n}\right| \leq$ $\left|d_{n}\right|+(C+1)\left|a_{n}\right|$ follows by the definition of $l\left(P_{1}\right)$. 


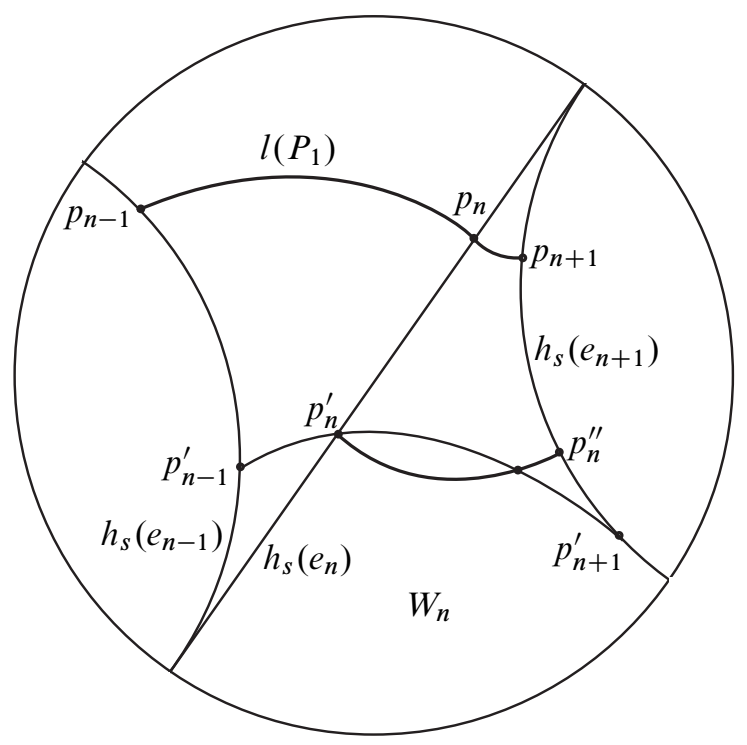

Figure 2: The points $P_{n}, P_{n}^{\prime}$ and $P_{n}^{\prime \prime}$

The above two estimates show $\left|d_{n}\right| \leq\left|d_{1}\right|+(C+1) \sum_{n \in \mathbf{N}}\left|a_{n}\right| \leq\left|d_{1}\right|+(C+1)|a|<\infty$. This implies that $l\left(P_{1}\right)$ stays a bounded distance from $a$. Thus the length of $l\left(P_{1}\right) \cap W_{n}$ and the length $a_{n}$ are comparable to a multiplicative constant. Therefore $l\left(P_{1}\right)$ has finite length which is a contradiction.

To find a necessary and sufficient condition on a shear function $s: \mathcal{F} \rightarrow \mathbf{R}$ such that $h_{s}$ has continuous extension to $x$, we compute the length of the above leaf $l\left(P_{1}\right)$ in terms of the shear function $s: \mathcal{F} \rightarrow \mathbf{R}$. Let $l_{n}$ be the length of the horocyclic $\operatorname{arc} l\left(P_{1}\right) \cap W_{n}$ in the wedge $W_{n}$ between $h_{s}\left(e_{n}\right)$ and $h_{s}\left(e_{n+1}\right)$. If $e_{n}, e_{n+1}$ and $e_{n+2}$ share a common endpoint, then an elementary hyperbolic geometry and the definition of $H_{s}$ show that the length of $l\left(P_{1}\right) \cap W_{n+1}$ in the wedge $W_{n+1}$ bounded by $H_{s}\left(e_{n+1}\right)$ and $H_{S}\left(e_{n+2}\right)$ is $l_{n} e^{s\left(e_{n+1}\right)}$ if $e_{n+1}<e_{n+2}$, and the length is $l_{n} e^{-s\left(e_{n+1}\right)}$ if $e_{n+2}<e_{n+1}$. If $e_{n}$, $e_{n+1}$ and $e_{n+2}$ do not share a common endpoint, then the length of $l\left(P_{1}\right) \cap W_{n+1}$ in the wedge $W_{n+1}$ between $H_{S}\left(e_{n+1}\right)$ and $H_{S}\left(e_{n+2}\right)$ is $l_{n}^{-1} e^{s\left(e_{n+1}\right)}$ if $e_{n+1}<e_{n+2}$, and the length is $l_{n}^{-1} e^{-s\left(e_{n+1}\right)}$ if $e_{n+1}>e_{n+2}$. Recall that $P_{1} \in h_{s}\left(e_{1}\right)$ is chosen such that $l_{1}=e^{s_{1}^{1}}$.

We show that

$$
l_{n}=e^{s_{1}^{n}+s_{2}^{n}+\cdots+s_{n}^{n}}
$$

by induction which finishes the proof. Assume that

$$
l_{n}=e^{s_{1}^{n}+s_{2}^{n}+\cdots+s_{n}^{n}}
$$


and we need to show that

$$
l_{n+1}=e^{s_{1}^{n+1}+s_{2}^{n+1}+\cdots+s_{n+1}^{n+1}} .
$$

We consider four possibilities and argue each separately. Assume first that $e_{n}, e_{n+1}$ and $e_{n+2}$ share a common endpoint and that $e_{n+1}<e_{n+2}$. Then

$$
l_{n+1}=l_{n} e^{s\left(e_{n+1}\right)}=e^{s_{1}^{n}+\cdots+s_{n}^{n}+s_{n+1}^{n+1}} .
$$

Since there is no additional change of fans from $e_{n+1}$ to $e_{n+2}$, we have $s_{i}^{n}=s_{i}^{n+1}$ for $i=1,2, \ldots, n$. This proves the formula in this case. The second case is when $e_{n}$, $e_{n+1}$ and $e_{n+2}$ share a common endpoint and $e_{n+2}<e_{n+1}$. Then we have

$$
l_{n+1}=l_{n} e^{-s\left(e_{n+1}\right)}=l_{n} e^{s_{n+1}^{n+1}}
$$

by the definition of $s_{n+1}^{n+1}$. The desired formula follows as in the previous case. In the third case we assume that $e_{n}, e_{n+1}$ and $e_{n+2}$ do not share a common endpoint and that $e_{n+1}<e_{n+2}$. Then

$$
l_{n+1}=l_{n}^{-1} e^{s\left(e_{n+1}\right)}=e^{-s_{1}^{n}-\cdots-s_{n}^{n}+s_{n+1}^{n+1}} .
$$

Since we have one additional change of fan from $e_{n+1}$ to $e_{n+2}$, we get that $s_{i}^{n+1}=-s_{i}^{n}$ for $i=1,2, \ldots, n$. This proves the formula in the third case. Finally, we assume that $e_{n}, e_{n+1}$ and $e_{n+2}$ do not share a common endpoint and that $e_{n+2}<e_{n+1}$. Then

$$
l_{n+1}=l_{n}^{-1} e^{-s\left(e_{n+1}\right)}=e^{-s_{1}^{n}-\cdots-s_{n}^{n}+s_{n+1}^{n+1}} .
$$

As in the previous case this gives the desired formula. Therefore the series

$$
\sum_{n=1}^{\infty} e^{s_{1}^{n}+\cdots+s_{n}^{n}}
$$

is the length of $l\left(P_{1}\right)$ and the proof of Theorem $\mathrm{C}$ is completed.

\section{Quasisymmetric maps and shears}

In this section we characterize shear maps which give rise to quasisymmetric maps of $\widehat{\mathbf{R}}$. This is the main result of the paper and, to our best knowledge, it gives the only known parametrization of the universal Teichmüller space $T(\mathbf{H})$.

Proof of the first part of Theorem A We prove that the first condition in the theorem is necessary for $s: \mathcal{F} \rightarrow \mathbf{R}$ to be a shear map of a quasisymmetric map $h: \widehat{\mathbf{R}} \rightarrow \widehat{\mathbf{R}}$.

Consider a fan of $\mathcal{F}$ with tip $p \in \hat{\mathbf{Q}}$. Let $A \in \operatorname{PSL}_{2}(\mathbf{Z})$ be such that $A(p)=\infty$. Let $B \in \mathrm{PSL}_{2}(\mathbf{R})$ be such that $B(h(p))=\infty$. Then $B \circ h \circ A^{-1}$ fixes $\infty$ and the 
corresponding shear function is $s \circ A^{-1}$. Moreover, if $h$ is $M_{1}$-quasisymmetric then $B \circ h \circ A^{-1}$ is $M$-quasisymmetric, where $M$ is a function of $M_{1}$ and is independent of $A$ and $B$. Therefore, we can study properties of a shear function on a single fan of $\mathcal{F}$ with tip $p$ by studying properties on the fan of $\mathcal{F}$ with tip $\infty$.

Consider an $M$-quasisymmetric map $h$ of $\widehat{\mathbf{R}}$ which fixes $\infty$ and let $s: \mathcal{F} \rightarrow \mathbf{R}$ be the induced shear function. Then $h$ satisfies

$$
\frac{1}{M} \leq \frac{h(n+k)-h(n)}{h(n)-h(n-k)} \leq M
$$

for all $n, k \in \mathbf{Z}$ with $k \neq 0$. This is the $M$-quasisymmetric condition taken at special symmetric triples in $\mathbf{Z} \subset \mathbf{R}$. We can further normalize $h$ by postcomposing with an affine map such that it fixes $n, n+1$ and $\infty$. The values at $\mathbf{Z}$ of such a normalized $h$ are uniquely determined by shears on the fan of $\mathcal{F}$ with tip $\infty$ by the definition of the characteristic map.

Let $e_{n}$ be the geodesic with endpoints $n$ and $\infty$, and let $s_{n}=s\left(e_{n}\right)$ for the convenience of notation. The condition (4) is equivalent to

$$
\frac{1}{M} \leq \frac{1+e^{s_{n+1}}+\cdots+e^{s_{n+1}+s_{n+2}+\cdots+s_{n+k-1}}}{e^{-s_{n}}+e^{-s_{n}-s_{n-1}}+\cdots+e^{-s_{n}-s_{n-1}-\cdots-s_{n-k+1}}} \leq M .
$$

The condition (5) is equivalent to the first condition in Theorem A and this establishes the necessity of the first condition in Theorem A.

We assume that a shear function $s: \mathcal{F} \rightarrow \mathbf{R}$ satisfies property (5) at each fan of $\mathcal{F}$ and show that characteristic map $h_{s}: \hat{\mathbf{Q}} \rightarrow \widehat{\mathbf{R}}$ extends to a quasisymmetric map of $\widehat{\mathbf{R}}$.

We first show that $h_{s}: \widehat{\mathbf{Q}} \rightarrow \widehat{\mathbf{R}}$ extends to a homeomorphism of $\widehat{\mathbf{R}}$. Since $h_{s}$ is a strictly monotone map of $\widehat{\mathbf{Q}}$ into $\widehat{\mathbf{R}}$, it is enough to show that $h_{s}(\widehat{\mathbf{Q}})$ is dense in $\widehat{\mathbf{R}}$. Assume on the contrary that $\widehat{\mathbf{R}} \backslash h_{S}(\widehat{\mathbf{Q}})$ contains an interval $I$. Assume that $I$ is a maximal such interval and let $l$ be the geodesic in $\mathbf{H}$ with endpoints equal to the endpoints of $I$. There are two possibilities to consider. Either $h_{s}(\hat{\mathbf{Q}})$ contains exactly one endpoint of $I$ or both endpoints of $I$ do not lie in $h_{s}(\widehat{\mathbf{Q}})$.

In the former case, the interval $I$ has an endpoint $h_{s}(p)$ for some $p \in \hat{\mathbf{Q}}$. This implies that the image of the fan at $p$ under $h_{s}$ accumulates to the geodesic $l \in \mathbf{H}$. Let $C$ be a horocycle based at $p$. Fix a single geodesic in the fan at $h_{s}(p)$. Then the sum of lengths of consecutive arcs of $C$ cut out by the geodesics in the fan at $h_{s}(p)$ which accumulate to $l$ starting from the fixed geodesic in the fan is finite. This implies that there exists a sequence of $2 n$ consecutive arcs on $C$ such that the ratio of the length of left $n$ consecutive arcs to the length of the right $n$ consecutive arcs is converging to $\infty$. Consequently, the condition (5) fails at the fan with tip $p$ which is a contradiction. 
In the later case, there is a sequence $e_{n} \in \mathcal{F}$ such that $h_{s}\left(e_{n}\right)$ converges to $l$ and that no $h_{s}\left(e_{n}\right)$ shares an endpoint with $l$. Moreover, we can assume that $\left\{e_{n}\right\}_{n \in \mathbf{N}}$ is a chain of geodesics in $\mathcal{F}$. We exhibit a sequence of pairs of adjacent triangles in $h(\mathcal{F})$ with shears converging to 0 or to $\infty$ which again contradicts condition (5). Let $e_{n_{0}}$ be such that $h_{s}\left(e_{n_{0}}\right)$ is close to $l$. Then $e_{n_{0}+1}$ shares an endpoint with $e_{n_{0}}$. Let $e_{n_{0}+k}$ be the geodesic in the chain $\left\{e_{n}\right\}_{n \in \mathbf{N}}$ with largest index which shares an endpoint with $e_{n_{0}}$. Then $e_{n_{0}+k+1}$ does not share an endpoint with $e_{n_{0}+k-1}$ (see Figure 3). We consider the two adjacent triangles in the complement of $\mathcal{F}$ with common boundary edge $e_{n_{0}+k}$. The image of the two triangles under $h_{s}$ has sides $h_{s}\left(e_{n_{0}+k-1}\right), h_{s}\left(e_{n_{0}+k}\right)$ and $h_{s}\left(e_{n_{0}+k+1}\right)$ close to the geodesic $l$. This implies that the other two sides are small in the Euclidean sense. Thus the shear is very large or very small which is a contradiction with condition (5). We proved that $h_{s}$ extends to a homeomorphism of $\hat{\mathbf{R}}$.

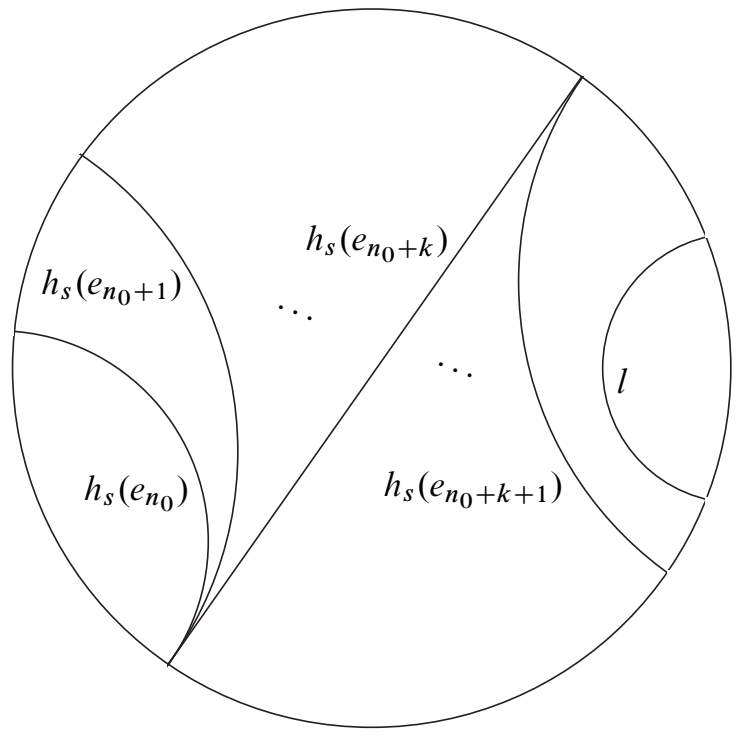

Figure 3: The accumulation to $l$

It remains to show that $h_{s}: \hat{\mathbf{R}} \rightarrow \widehat{\mathbf{R}}$ is a quasisymmetric map. Let $F_{s}=\operatorname{ex}\left(h_{s}\right)$ be the barycentric extension of $h_{s}$ (see Douady and Earle [5] for the definition). Then $F_{s}: \mathbf{H} \rightarrow \mathbf{H}$ is a real analytic diffeomorphism of $\mathbf{H}$. The map $h_{s}$ is quasisymmetric if and only if $F_{S}$ is quasiconformal. Let $\mu_{F_{S}}=\bar{\partial} F_{S} / \partial F_{S}$ be the Beltrami coefficient of $F_{s}$.

Assume on the contrary that $F_{S}$ is not quasiconformal. Then there exists a sequence $z_{n} \in \mathbf{H}$ such that $\left|\mu_{F_{S}}\left(z_{n}\right)\right| \rightarrow 1$ as $n \rightarrow \infty$. Since $F_{S}$ is a real analytic diffeomorphism 
(see [5]), it follows that $z_{n}$ leaves every compact subset of $\mathbf{H}$. There are two possibilities for $z_{n}$. Either there exist a horoball $D$ with center at $\infty$ and a subsequence $z_{n_{k}}$ of $z_{n}$ such that $z_{n_{k}}$ lies outside the $\operatorname{PSL}_{2}(\mathbf{Z})$ orbit of $D$, or sequence $z_{n}$ enters the $\operatorname{PSL}_{2}(\mathbf{Z})$ orbit of every horoball with center at $\infty$.

Suppose that we are in the former case. For simplicity, denote the subsequence $z_{n_{k}}$ by $z_{n}$ again. Let $\Delta_{n}$ be triangle in the complement of $\mathcal{F}$ which contains $z_{n}$. Let $A_{n} \in \mathrm{PSL}_{2}(\mathbf{Z})$ be such that $A_{n}\left(\Delta_{n}\right)=\Delta_{0}$. Let $B_{n} \in \mathrm{PSL}_{2}(\mathbf{R})$ be such that $B_{n} \circ h_{s} \circ A_{n}^{-1}$ fixes 0,1 and $\infty$. By the conformal naturality of the barycentric extension, we have that

$$
\operatorname{ex}\left(B_{n} \circ h_{s} \circ A_{n}^{-1}\right)=B_{n} \circ F_{s} \circ A_{n}^{-1}=F_{n} .
$$

Let $z_{n}^{\prime}=A_{n}\left(z_{n}\right) \in \Delta_{0}$. Then $z_{n}^{\prime}$ belongs to a compact subset of $\mathbf{H}$ and $\left|\mu_{F_{n}}\left(z_{n}^{\prime}\right)\right|=$ $\left|\mu_{F_{s}}\left(z_{n}\right)\right|$. Condition (5) implies that individual shears are bounded by $1 / M$ from below and by $M$ from above. This implies that the sequence of shear maps $s \circ A_{n}^{-1}$ corresponding to homeomorphisms $B_{n} \circ h_{s} \circ A_{n}^{-1}$ has a convergent subsequence in the sense that for each edge $e \in \mathcal{F}$ the sequence of real numbers $s \circ A_{n_{k}}^{-1}(e)$ converges as $k \rightarrow \infty$. The limiting map $s_{\infty}: \mathcal{F} \rightarrow \mathbf{R}$ satisfies property (5) in each fan with the constant $M$ because each $s \circ A_{n}^{-1}$ does. By the normalization of $B_{n} \circ h_{s} \circ A_{n}^{-1}$, we get that $B_{n_{k}} \circ h_{s} \circ A_{n_{k}}^{-1}$ pointwise converges to a homeomorphism $h_{s_{\infty}}$ of $\hat{\mathbf{R}}$ with shear map $s_{\infty}$. By the continuity of the barycentric extension, we get that $\left|\mu_{F_{n_{k}}}\right|$ converges to $\left|\mu_{\mathrm{ex}\left(h_{s \infty}\right)}\right|$ uniformly on compact subsets of $\mathbf{H}$. This implies that for a compact subset $K$ of $\mathbf{H}$ there exists $a<1$ such that $\left|\mu_{F_{n_{k}}}\right| \leq a$ on $K$. On the other hand, we have that $\left|\mu_{F_{n_{k}}}\left(z_{n_{k}}\right)\right| \rightarrow 1$ as $k \rightarrow \infty$ which gives a contradiction.

Suppose that we are in the later case. Namely, $\left|\mu_{F_{s}}\left(z_{n}\right)\right| \rightarrow 1$ as $n \rightarrow \infty$ with $z_{n}$ entering the $\mathrm{PSL}_{2}(\mathbf{Z})$ orbit of every horoball based at $\infty$. Let $\Delta_{n}$ be a complementary triangle of $\mathcal{F}$ which contains $z_{n}$. Let $A_{n} \in \mathrm{PSL}_{2}(\mathbf{Z})$ be such that $A_{n}\left(\Delta_{n}\right)=\Delta_{0}$ and that $A_{n}\left(z_{n}\right)=z_{n}^{\prime} \rightarrow \infty$ as $n \rightarrow \infty$. Let $B_{n} \in \mathrm{PSL}_{2}(\mathbf{R})$ be such that $B_{n} \circ h_{s} \circ A_{n}^{-1}=h_{n}$ fixes 0,1 and $\infty$. Then $h_{n}$ satisfies property (5) with the same constant $M$ as does $h$. By the conformal naturality of the barycentric extension, $\left|\mu_{F_{s}}\left(z_{n}\right)\right|=\left|\mu_{\mathrm{ex}\left(h_{n}\right)}\left(z_{n}^{\prime}\right)\right| \rightarrow 1$ as $n \rightarrow \infty$. Let $\lambda_{n}=\operatorname{Im}\left(z_{n}^{\prime}\right)$ and let $\lambda_{n}^{\prime}$ be such that $\widehat{h}_{n}(x)=\left(1 / \lambda_{n}^{\prime}\right) h_{n}\left(\lambda_{n} x\right)$ fixes 1 . It is clear that $\hat{h}_{n}$ fixes 0 and $\infty$ as well. Let $w_{n}=\left(1 / \lambda_{n}\right) z_{n}^{\prime}$. Then $w_{n} \rightarrow i$ and $\left|\mu_{\mathrm{ex}\left(\hat{h}_{n}\right)}\left(w_{n}\right)\right|=\left|\mu_{\mathrm{ex}\left(h_{n}\right)}\left(z_{n}^{\prime}\right)\right|=\left|\mu_{F_{s}}\left(z_{n}\right)\right| \rightarrow 1$ as $n \rightarrow \infty$. We need the following lemma in order to finish the proof.

Lemma 5.1 Under the above normalization, there exists a constant $c_{0}>1$ such that $1 / c_{0} \leq-\widehat{h}_{n}(-1) \leq c_{0}$.

Proof Let $k_{n} \in \mathbf{N}$ be such that $k_{n} \leq \lambda_{n} \leq k_{n}+1$. Then $h_{n}\left(k_{n}\right) \leq h_{n}\left(\lambda_{n}\right)=\lambda_{n}^{\prime} \leq$ $h_{n}\left(k_{n}+1\right)$. By property (5), we have that $h_{n}\left(k_{n}+1\right)-h_{n}\left(k_{n}\right) \leq M h_{n}\left(k_{n}\right)$. This 
implies that

$$
h_{n}\left(k_{n}+1\right) \leq(M+1) h_{n}\left(k_{n}\right) \leq(M+1) \lambda_{n}^{\prime} .
$$

By applying property (5) to $h_{n}$ at points $-\left(k_{n}+1\right), 0$ and $k_{n}+1$, we get that $(1 / M) h_{n}\left(k_{n}+1\right) \leq-h_{n}\left(-k_{n}-1\right) \leq M h_{n}\left(k_{n}+1\right)$. Similarly, $(1 / M) h_{n}\left(k_{n}\right) \leq$ $-h_{n}\left(-k_{n}\right) \leq M h_{n}\left(k_{n}\right)$. These two inequalities imply that

$$
-M h_{n}\left(k_{n}+1\right) \leq h_{n}\left(-k_{n}-1\right) \leq h_{n}\left(-\lambda_{n}\right) \leq h_{n}\left(-k_{n}\right) \leq-\frac{1}{M} h_{n}\left(k_{n}\right) \text {. }
$$

From (6), we get

$$
h_{n}\left(k_{n}\right) \geq \frac{1}{M+1} h_{n}\left(k_{n}+1\right) \geq \frac{1}{M+1} \lambda_{n}^{\prime} .
$$

The above two inequalities and (6) give that

$$
-M(M+1) \lambda_{n}^{\prime} \leq h_{n}\left(-\lambda_{n}\right) \leq-\frac{1}{M(M+1)} \lambda_{n}^{\prime}
$$

which implies

$$
-M(M+1) \leq \frac{1}{\lambda_{n}^{\prime}} h_{n}\left(-\lambda_{n}\right)=\hat{h}_{n}(-1) \leq-\frac{1}{M(M+1)} .
$$

Take $c_{0}=M(M+1)$ and the above becomes $1 / c_{0} \leq-\widehat{h}_{n}(-1) \leq c_{0}$.

We finish the proof using the above lemma. Note that $\widehat{h}_{n}$ fixes 0,1 and $\infty$, and $\widehat{h}_{n}(-1)$ is bounded away from 0 and $\infty$ by the above lemma. Then Lemma 2.2 implies that $\left|\mu \hat{h}_{n}\right| \leq c<1$ in a neighborhood of $i \in \mathbf{H}$ and for all $n \in \mathbf{N}$ (see also [13, Lemma 3.6], [1], [5]). On the other hand, the assumption on $w_{n}$ and conformal naturality of barycentric extension implies that $\left|\mu \hat{h}_{n}\left(w_{n}\right)\right| \rightarrow 1$ as $n \rightarrow \infty$ which is a contradiction. This finishes the proof of the first statement in Theorem A.

Proof of the second part of Theorem A Consider a fan of geodesics $\left\{e_{n}\right\}_{n \in \mathbf{N}}$ of $\mathcal{F}$ with tip $p \in \widehat{\mathbf{Q}}$. Let $a_{n} \in \widehat{\mathbf{Q}}$ be the endpoint of $e_{n}$ that is different from $p$. Then $\left(a_{k}, a_{m}, a_{n}, p\right)$ are in the cyclic order of $\widehat{\mathbf{R}}$ if $k<m<n$. The triple $a_{k}, a_{m}, a_{n}$ is said to be fan-symmetric if $m-k=n-m$. The point $a_{m}$ is said to be the midpoint of the triple.

Let $a_{k}, a_{m}, a_{n} \in \widehat{\mathbf{Q}}$ be a fan-symmetric triple for the fan with tip $p \in \widehat{\mathbf{Q}}$, where $a_{m}$ is the mid-point of the triple. This implies that

$$
\operatorname{cr}\left(p, a_{k}, a_{m}, a_{n}\right)=\frac{\left(a_{m}-p\right)\left(a_{n}-a_{k}\right)}{\left(a_{n}-p\right)\left(a_{m}-a_{k}\right)}=2 .
$$


The Farey generation of a triple $\left(e_{k}, e_{m}, e_{n}\right)$ of geodesics with $m-k=n-m$ is the minimum of the Farey generations of $e_{k}$ and $e_{n}$. Let $h: \hat{\mathbf{R}} \rightarrow \widehat{\mathbf{R}}$ be a symmetric map which fixes 0,1 and $\infty$. If the generation of a triple $e_{k}, e_{m}, e_{n}$ is large, it follows that the points $a_{k}, p$ and $a_{n}$ are close in the angle metric of $\widehat{\mathbf{R}}$ with respect to $i \in \mathbf{H}$. The barycentric extension ex $(h)=F$ of $h$ has Beltrami coefficient close to zero in a definite Euclidean neighborhood in $\mathbf{H}$ of the triple $\left(a_{k}, p, a_{n}\right)$ (see [7]). A length-area argument implies that $\operatorname{cr}\left(h(p), h\left(a_{k}\right), h\left(a_{m}\right), h\left(a_{n}\right)\right)$ is close to 2 (see, for example, [10]). After postcomposing $h$ by $A \in \mathrm{PSL}_{2}(\mathbf{R})$ such that $A \circ h\left(a_{m}\right)=\infty$, this is equivalent to the fact that the ratio

$$
\frac{\left|A \circ h(p)-A \circ h\left(a_{k}\right)\right|}{\left|A \circ h\left(a_{n}\right)-A \circ h(p)\right|}
$$

is close to 1 . Let $s: \mathcal{F} \rightarrow \mathbf{R}$ be the shear map of $h$ and let $s_{i}=s\left(e_{i}\right)$. Then for a given $\epsilon>0$, there exists $k=k(\epsilon) \in \mathbf{N}$ such that on any fan-symmetric triple of generation at least $k$ the shear map $s: \mathcal{F} \rightarrow \mathbf{R}$ satisfies

$$
\frac{1}{1+\epsilon} \leq \frac{1+e^{s_{m+1}}+\cdots+e^{s_{m+1}+s_{m+2}+\cdots+s_{n}}}{e^{-s_{m}}+e^{-s_{m}-s_{m-1}}+\cdots+e^{-s_{m}-s_{m-1}-\cdots-s_{k}}} \leq 1+\epsilon .
$$

Thus we established the necessity of the second condition in Theorem A.

We show that the second condition in Theorem A is also sufficient for a map to be symmetric. For any $k \in \mathbf{N}$, there are only finitely many geodesics in $\mathcal{F}$ whose generation is at most $k$. Together with (7), this implies that the shear function $s: \mathcal{F} \rightarrow \mathbf{R}$ is bounded and that $s(e)$ converges to 0 as the generation of $e$ converges to $\infty$, where the speed of convergence depends only on the generation of $e \in \mathcal{F}$. The characteristic map $h_{s}$ of the shear map $s$ with property (7) extends to a homeomorphism of $\hat{\mathbf{R}}$. The proof follows the same lines as in the proof of the first part of Theorem A and we omit it here.

It remains to show that $h_{s}$ is a symmetric map. We consider the barycentric extension $\operatorname{ex}\left(h_{s}\right)=F_{s}$ of $h_{s}$. It is enough to show that $F_{S}$ is an asymptotically conformal map of $\mathbf{H}$ (see [7]).

Assume on the contrary that there exists a sequence $z_{n} \in \mathbf{H}$ which leaves every compact subset of $\mathbf{H}$ such that $\left|\mu_{F_{s}}\left(z_{n}\right)\right| \geq c>0$. Let $\Delta_{n}$ be the ideal triangle in the complement of $\mathcal{F}$ which contains $z_{n}$. Let $A_{n} \in \mathrm{PSL}_{2}(\mathbf{Z})$ be such that $A_{n}\left(\Delta_{n}\right)=\Delta_{0}$, where $\Delta_{0}$ is the triangle in $\mathcal{F}$ with vertices 0,1 and $\infty$. Let $B_{n} \in \mathrm{PSL}_{2}(\mathbf{R})$ be such that $h_{n}=B_{n} \circ h_{s} \circ A_{n}^{-1}$ fixes 0,1 and $\infty$. Let $z_{n}^{\prime}=A_{n}\left(z_{n}\right) \in \Delta_{0}$ and let $F_{n}=\operatorname{ex}\left(h_{n}\right)$ be the barycentric extension of $h_{n}$.

Assume first that a subsequence of $z_{n}^{\prime}$ stays in a compact part of $\Delta_{0}$, and for simplicity we denote the subsequence by $z_{n}^{\prime}$ again. This implies that the sequence of $\Delta_{n}$ 's 
contains infinitely many pairwise different triangles because $z_{n}$ leave any compact subset of $\mathbf{H}$. In particular, the minimum of the generations of the boundary geodesics of $\Delta_{n}$ converges to infinity as $n \rightarrow \infty$. Consequently, shear maps $s \circ A_{n}^{-1}$ converge to the zero map which implies that $h_{n}$ converges pointwise on $\widehat{\mathbf{R}}$ to the identity. On the other hand, $\left|\mu_{F_{n}}\left(z_{n}^{\prime}\right)\right| \geq c>0$ by conformal naturality of the barycentric extension. This is a contradiction with the continuity properties of the barycentric extension (see [5] or Section 2).

In the other case, we assume that $z_{n}^{\prime} \rightarrow \infty$ inside $\Delta_{0}$ as $n \rightarrow \infty$. Let $\lambda_{n, 1}=\left[\operatorname{Im}\left(z_{n}^{\prime}\right)\right]$ be the greatest integer less than or equal to $\operatorname{Im}\left(z_{n}^{\prime}\right)$. Clearly $\lambda_{n, 1} \rightarrow \infty$ as $n \rightarrow \infty$. Let $\lambda_{n, 1}^{\prime}=h_{n}\left(\lambda_{n, 1}\right)$. Define $\left(1 / \lambda_{n, 1}^{\prime}\right) h_{n}\left(\lambda_{n, 1} x\right)=\widetilde{h}_{n, 1}(x)$ and note that $\widetilde{h}_{n, 1}(x)$ fixes 0 , 1 and $\infty$.

We show that $\lim _{n \rightarrow \infty} \widetilde{h}_{n, 1}(k)=k$ for all $k \in \mathbf{Z}$ except possibly one integer $k=k_{0}$. A basic observation is that for at most one integer $k_{0} \in \mathbf{Z}$ we have that the Farey generation of $e_{\lambda_{n, 1} k_{0}}$ is bounded as $n \rightarrow \infty$. Indeed, if the Farey generation of $e_{\lambda_{n, 1} k_{0}}$ is bounded then for $k \neq k_{0}$ we have $\left|\lambda_{n, 1} k_{0}-\lambda_{n, 1} k\right| \rightarrow \infty$ as $n \rightarrow \infty$ (because $\lambda_{n, 1} \rightarrow \infty$ as $n \rightarrow \infty$.) Thus, in the fan $\left\{e_{k}^{n, 1}\right\}_{k \in \mathbf{Z}}$ with the tip $\left(h_{n}\right)^{-1}(\infty)$ there is $\lambda_{n, 1}\left|k-k_{0}\right|$ geodesics separating $e_{\lambda_{n, 1} k}$ and $e_{\lambda_{n, 1} k_{0}}$. Since the Farey generation of $e_{\lambda_{n, 1} k_{0}}$ is bounded it follows that the Farey generation of $e_{\lambda_{n, 1} k}$ is unbounded.

Assume that the Farey generations of $e_{\lambda_{n, 1} k}$ go to infinity as $n \rightarrow \infty$ for all $k \in \mathbf{Z} \backslash\left\{k_{0}\right\}$. We consider three cases: $k_{0}=0, k_{0}<0$ and $k_{0}>0$. If $k_{0}=0$ then $\tilde{h}_{n, 1}(-1) \rightarrow-1$ as $n \rightarrow \infty$ because condition (7) gives

$$
\frac{\tilde{h}_{n, 1}(1)-\tilde{h}_{n, 1}(0)}{\widetilde{h}_{n, 1}(0)-\widetilde{h}_{n, 1}(-1)} \rightarrow 1
$$

as $n \rightarrow \infty$, and $\tilde{h}_{n, 1}(1)=1, \tilde{h}_{n, 1}(0)=0$. By a similar use of symmetric triple $(-1,1,3)$ we obtain that $\widetilde{h}_{n, 1}(3) \rightarrow 3$ as $n \rightarrow \infty$ because $\widetilde{h}_{n, 1}(1)=1$ and $\widetilde{h}_{n, 1}(-1) \rightarrow-1$ as $n \rightarrow \infty$. Then we use $(1,2,3)$ to obtain $\tilde{h}_{n, 1}(2) \rightarrow 2$ as $n \rightarrow \infty$. Next we use triples $(2,3,4),(3,4,5), \ldots$ in a similar procedure to obtain that $\tilde{h}_{n, 1}(k) \rightarrow k$ as $n \rightarrow \infty$ for all $k \in \mathbf{N}$. After that, use the triples $(-k, 0, k)$ for $k \in \mathbf{N}$ to conclude that $\widehat{h}_{n, 1}(k) \rightarrow k$ as $n \rightarrow \infty$ for all $k \in \mathbf{Z}$. (We used the fact that if $(x, y, z)$ is a symmetric triple in $\mathbf{Z}$ such that the Farey generations of $e_{x}^{n, 1}$ and $e_{z}^{n, 1}$ go to infinity as $n \rightarrow \infty$ and if $\tilde{h}_{n, 1}$ converges to the identity on any two of the points of the triple $(x, y, z)$, then $\widetilde{h}_{n, 1}$ converges to the identity on the third point of the triple.) If $k_{0}<0$ then we use $(0,1,2)$ to conclude that $\tilde{h}_{n, 1}(2) \rightarrow 2$ as $n \rightarrow \infty$. Further we use $(1,2,3),(2,3,4), \ldots$ to conclude that $\tilde{h}_{n, 1}(k) \rightarrow k$ as $n \rightarrow \infty$ for all $k \in \mathbf{N}$. Then we use triples $(k, 0,-k)$ for all $k<0$ and $k \neq k_{0}$ to conclude that $\tilde{h}_{n, 1}(k) \rightarrow k$ as $n \rightarrow \infty$ for all $k \in \mathbf{Z} \backslash\left\{k_{0}\right\}$. The proof for $k_{0}>0$ is similar. 
Let $\lambda_{n, r}$ be the greatest integer multiple of $2^{r-1}$ which is less than or equal to $\operatorname{Im}\left(z_{n}^{\prime}\right)$ for fixed $r \in \mathbf{N}$. Clearly $\lambda_{n, r} \rightarrow \infty$ as $n \rightarrow \infty$. Let $\lambda_{n, r}^{\prime}=h_{n}\left(\lambda_{n, r}\right)$. Define $\left(1 / \lambda_{n, r}^{\prime}\right) h_{n}\left(\lambda_{n, r} x\right)=\widetilde{h}_{n, r}(x)$ and note that $\tilde{h}_{n, r}(x)$ fixes 0,1 and $\infty$. We claim that $\lim _{n \rightarrow \infty} \widetilde{h}_{n, r}\left(k / 2^{i}\right)=k / 2^{i}$ for all $k \in \mathbf{Z} \backslash\left\{k_{0}\right\}$ and $i=0, \ldots, r-1$, where $k_{0}$ is fixed. The proof is by finite induction on $i$. The case $i=0$ is proved in the above paragraph. Assume that the statement is true for $i$ and we need to prove that it is true for $i+1$. The inductive hypothesis says that $\lim _{n \rightarrow \infty} \tilde{h}_{n, r}\left(k / 2^{i}\right)=k / 2^{i}$ for $k \in \mathbf{Z}$ because $\lambda_{n, r}\left(k / 2^{i}\right) \in \mathbf{Z}$ for each $n \in \mathbf{N}$. Since each $k / 2^{i+1}$, for $k \in \mathbf{Z}$ odd, is in the middle of $(k-1) / 2^{i+1}$ and $(k+1) / 2^{i+1}$ on which the convergence holds and since $\lambda_{n, r}\left(k / 2^{i+1}\right) \in \mathbf{Z}$, it follows similar to the above that $\lim _{n \rightarrow \infty} \tilde{h}_{n, r}\left(k / 2^{i+1}\right)=k / 2^{i+1}$. We note that among all $k / 2^{r}$ for $k \in \mathbf{Z}$ there is at most one $k_{0} / 2^{r}$ such that we cannot conclude that $\hat{h}_{n, 1}\left(k_{0} / 2^{r}\right) \rightarrow k_{0} / 2^{r}$ as $n \rightarrow \infty$. This finishes the induction.

We use the Cantor diagonalization process to obtain a contradiction. The set $D=$ $\left\{k / 2^{r-1}: r \in \mathbf{N}, k \in \mathbf{Z}\right\}$ is a dense subset of $\widehat{\mathbf{R}}$. We put $D$ into a sequence $\left\{b_{m}\right\}$ such that if $b_{m}=k / 2^{r-1}$ for minimal $r \in \mathbf{N}$ then $m \geq r$. Fix $m \in \mathbf{N}$. Then there exists $n_{m}$ such that $\left|\tilde{h}_{n_{m}, m}\left(b_{i}\right)-b_{i}\right|<1 / m$ for $i=1,2, \ldots, m$ and $\left|z_{m}^{\prime} / \lambda_{n_{m}, m}-i\right|<1 / m$. This implies that $\widetilde{h}_{n_{m}, m}$ converges pointwise to the identity on $\widehat{\mathbf{R}}$. On the other hand, the Beltrami coefficient of $\operatorname{ex}\left(\widetilde{h}_{n_{m}, m}\right)$ at $z_{m}^{\prime} / \lambda_{n_{m}, m}$ is bounded away from 0 by conformal naturality of the barycentric extension. This is a contradiction. Therefore $h_{s}$ is symmetric which finishes the proof of Theorem A.

\section{The topology on $\mathcal{X}$}

Let $\mathcal{X}$ be the space of all shear functions $s: \mathcal{F} \rightarrow \mathbf{R}$ which satisfy condition (5) on each fan of geodesics in $\mathcal{F}$ with the same constant. Theorem A implies that the universal Teichmüller space $T(\mathbf{H})$ is parameterized by the space $\mathcal{X}$. We turn our attention to finding a topology on $\mathcal{X}$ which would make the map $T(\mathbf{H}) \rightarrow \mathcal{X}$ a homeomorphism.

Consider a shear function $s \in \mathcal{X}$ and a fan of geodesics $\left\{e_{n}\right\}_{n \in \mathbf{Z}}$ of $\mathcal{F}$ with tip $p$. For a given horocycle $C$ with center $p$, we denote by $s(p ; m, k)$ the quotient of the length of arc of $C$ between $h_{s}\left(e_{m+k+1}\right)$ and $h_{s}\left(e_{m}\right)$ to the length of the arc of $C$ between $h_{s}\left(e_{m}\right)$ and $h_{s}\left(e_{m-k-1}\right)$, for $m, k \in \mathbf{Z}$ and $k \geq 0$. Recall that $s(p ; m, k)$ is the expression in the middle of (5) described in a coordinate independent fashion.

Let $M(s) \geq 1$ be the supremum of $s(p ; m, k)$ over all $p \in \widehat{\mathbf{Q}}, m, k \in \mathbf{Z}$. If $M(s)<\infty$, then we say that $s: \mathcal{F} \rightarrow \mathbf{R}$ satisfies $M(s)$-condition at the fan with tip $p$. For example, the shear map $s_{\mathrm{id}}$ of the basepoint id $\in T(\mathbf{H})$ is assigning 0 to each edge of $\mathcal{F}$ and $M\left(s_{\text {id }}\right)=1$. 
More generally, let $s_{1}, s_{2} \in \mathcal{X}$. Define $M\left(s_{1}, s_{2}\right)$ to be the supremum of the maximum of $s_{1}(p ; m, k) / s_{2}(p ; m, k)$ and $s_{2}(p ; m, k) / s_{1}(p ; m, k)$ over all $p \in \widehat{\mathbf{Q}}$ and $m, k \in \mathbf{Z}$. Note that $M\left(s_{1}, s_{2}\right)=M\left(s_{2}, s_{1}\right)$ and that $M\left(s_{1}, s_{\text {id }}\right)=M\left(s_{1}\right)$.

Let $h_{n}: \hat{\mathbf{R}} \rightarrow \widehat{\mathbf{R}}$ be a sequence of quasisymmetric maps which fix 0,1 and $\infty$, and which converge to the identity in the Teichmüller topology in $T(\mathbf{H})$. Then we immediately obtain that $M\left(s_{h_{n}}\right) \rightarrow 1$ as $n \rightarrow \infty$ from the quasisymmetric condition.

Proof of Theorem B Recall that $h_{n} \rightarrow$ id in the Teichmüller topology if and only if

$$
\sup \frac{\operatorname{cr}\left(h_{n}(a), h_{n}(b), h_{n}(c), h_{n}(d)\right)}{\operatorname{cr}(a, b, c, d)} \rightarrow 1
$$

as $n \rightarrow \infty$, where the cross-ratio is

$$
\operatorname{cr}(a, b, c, d)=\frac{(c-a)(d-b)}{(d-a)(c-b)}
$$

and the supremum is over all quadruples $(a, b, c, d) \in(\widehat{\mathbf{R}})^{4}$ with the cross-ratio between $1+1 / M$ and $1+M$ for some $M>1$. By the definition, $h_{n} \rightarrow h$ as $n \rightarrow \infty$ in the Teichmüller topology if and only if $h_{n} \circ h^{-1} \rightarrow$ id in the Teichmüller topology. A quadruple of points in $\widehat{\mathbf{Q}}$ with cross-ratio 2 where one point is the tip of the fan such that the other three points are endpoints of geodesics in the fan is fan-symmetric (see the proof of Theorem A for equivalent definition). The cross-ratio of the image under $h$ of a fan-symmetric quadruple is bounded away from 1 and $\infty$ because $h$ is quasisymmetric. The above characterization of the Teichmüller topology when applied to $h_{n} \circ h^{-1} \rightarrow$ id at the images under $h$ of all fan-symmetric quadruples gives that $M\left(s_{h_{n}}, s_{h}\right) \rightarrow 1$ as $n \rightarrow \infty$. This proves the necessity of the condition.

Given $h, h_{n} \in T(\mathbf{H})$ such that $M\left(s_{h_{n}}, s_{h}\right) \rightarrow 1$ as $n \rightarrow \infty$, we need to show that $h_{n} \rightarrow h$ as $n \rightarrow \infty$. Assume on the contrary that $h_{n}$ does not converge to $h$ in the Teichmüller topology. Let $F=\operatorname{ex}(h)$ and $F_{n}=\operatorname{ex}\left(h_{n}\right)$ be the barycentric extensions of $h$ and $h_{n}$, respectively. The assumption implies that there exists $c>0$ and a sequence $z_{n} \in \mathbf{H}$ such that $\left|\mu_{F}\left(z_{n}\right)-\mu_{F_{n}}\left(z_{n}\right)\right| \geq c$. There are two possibilities for the sequence $z_{n}$. Either there exists a horoball $C$ with center $\infty$ and a subsequence $z_{n_{k}}$ such that $z_{n_{k}}$ is disjoint from the $\mathrm{PSL}_{2}(\mathbf{Z})$ orbit of $C$, or for any horoball $C$ with center $\infty$ only finitely many $z_{n}$ 's lie outside the $\mathrm{PSL}_{2}(\mathbf{Z})$ orbit of $C$.

Assume we are in the former case. For the convenience of notation, replace $z_{n_{k}}$ with $z_{n}$. Let $A_{n} \in \mathrm{PSL}_{2}(\mathbf{Z})$ be such that $A_{n}\left(\Delta_{n}\right)=\Delta_{0}$, where $\Delta_{n}$ is a complementary triangle of $\mathcal{F}$ which contains $z_{n}$. Then $A_{n}\left(z_{n}\right)$ lies in a compact subset of $\mathbf{H}$. Let $B_{n}, B_{n}^{*} \in \mathrm{PSL}_{2}(\mathbf{R})$ be such that $B_{n} \circ h \circ A_{n}^{-1}$ and $B_{n}^{*} \circ h_{n} \circ A_{n}^{-1}$ fix 0,1 and $\infty$. Since $M\left(s_{h_{n}}, s_{h}\right) \rightarrow 1$ as $n \rightarrow \infty$, we get that $B_{n} \circ h \circ A_{n}^{-1}$ and $B_{n}^{*} \circ h_{n} \circ A_{n}^{-1}$ pointwise 
converge to the same quasisymmetric map. Therefore the Beltrami coefficients of their corresponding barycentric extensions converge uniformly on compact subsets of $\mathbf{H}$ to the same Beltrami coefficient (see [5]). This contradicts $\left|\mu_{F}\left(z_{n}\right)-\mu_{F_{n}}\left(z_{n}\right)\right| \geq c$.

Assume we are in the later case. Let $A_{n} \in \mathrm{PSL}_{2}(\mathbf{Z})$ and $B_{n}, B_{n}^{*} \in \mathrm{PSL}_{2}(\mathbf{R})$ be as above. Let $h_{n}^{\prime}=B_{n} \circ h \circ A_{n}^{-1}$ and $h_{n}^{*}=B_{n}^{*} \circ h_{n} \circ A_{n}^{-1}$. In addition, we may assume that $A_{n}\left(z_{n}\right) \rightarrow \infty$ as $n \rightarrow \infty$. To find a contradiction in this case, we use the idea from the proof of the last part of Theorem A. Denote by $\lambda_{n, r}$ the largest integer multiple of $2^{r-1}$ which is less than or equal to $\operatorname{Im}\left(z_{n}\right)$. Let $\lambda_{n, r}^{\prime}=h_{n}^{\prime}\left(\lambda_{n, r}\right)$ and $\lambda_{n, r}^{*}=h_{n}^{*}\left(\lambda_{n, r}\right)$. Then

$$
\tilde{h}_{n, r}^{\prime}(x)=\left(1 / \lambda_{n, r}^{\prime}\right) h_{n}^{\prime}\left(\lambda_{n, r} x\right) \quad \text { and } \quad \tilde{h}_{n, r}^{*}(x)=\left(1 / \lambda_{n, r}^{*}\right) h_{n}^{*}\left(\lambda_{n, r} x\right)
$$

fix 0,1 and $\infty$. For each $r \in \mathbf{N}$, the sequences $\widetilde{h}_{n, r}^{\prime}(x)$ and $\widetilde{h}_{n, r}^{*}(x)$ have convergent subsequences (in the pointwise sense) whose limits $h_{r}^{1}$ and $h_{r}^{2}$ agree on the set $\left\{k / 2^{r-1}: k \in \mathbf{Z}\right\}$ because $M\left(s_{h_{n}}, s_{h}\right) \rightarrow 1$ as $n \rightarrow \infty$. The values of maps $h_{r}^{1}$ and $h_{r}^{2}$ on $\left\{k / 2^{r-1}: k \in \mathbf{Z}\right\}$ depend only on the shears of $h_{n}^{\prime}$ and $h_{n}^{*}$ on the fan with tip $\infty$. Using the Cantor diagonalization process, we find sequences

$$
\left\{\tilde{h}_{n_{m}, m}^{\prime}(x)\right\}_{m} \text { and }\left\{\tilde{h}_{n_{m}, m}^{*}(x)\right\}_{m}
$$

whose pointwise limits $h^{1}$ and $h^{2}$ satisfy $h^{1}=h^{2}$ and $z_{m}^{\prime} / \lambda_{n_{m}, m} \rightarrow i \in \mathbf{H}$ as $m \rightarrow \infty$. This again gives a contradiction with $\left|\mu_{F}\left(z_{m}\right)-\mu_{F_{m}}\left(z_{m}\right)\right| \geq c$ by conformal naturality of the barycentric extension.

\section{Decorated tesselations and lambda lengths}

A tesselation $\tau$ of $\mathbf{H}$ is a locally finite ideal geodesic triangulation of $\mathbf{H}$ with a distinguished oriented geodesic. A decorated tesselation $\tilde{\tau}$ is a tesselation $\tau$ of $\mathbf{H}$ together with an assignment of a horocycle to each vertex of $\tau$ whose center is that vertex (see [15]).

Let $\tau$ be a tesselation with a distinguished oriented edge $e=\left(x_{i}, x_{t}\right)$, where $x_{i}$ is the initial point and $x_{t}$ is the terminal point of $e$. Recall that $\mathcal{F}$ is the Farey tesselation and let $(-1,1)$ be a distinguished oriented geodesic of $\mathcal{F}$. Denote by $\tau^{0}$ the set of vertices of $\tau$. Recall that $\widehat{\mathbf{Q}} \subset \widehat{\mathbf{R}}$ is the set of vertices of $\mathcal{F}$. There exists a unique map $h_{\tau}: \hat{\mathbf{Q}} \rightarrow \tau^{0}$ such that $h_{\tau}\left(x_{i}\right)=-1, h_{\tau}\left(x_{t}\right)=1$ and that if $x, y, x \in \widehat{\mathbf{Q}}$ are vertices of a complementary triangle of $\mathcal{F}$ then $h_{\tau}(x), h_{\tau}(y), h_{\tau}(z) \in \tau^{0}$ are the vertices of a complementary triangle of $\tau$ (see [15]). We call $h_{\tau}$ the characteristic map of $\tau$. It is clear that $h_{\tau}: \widehat{\mathbf{Q}} \rightarrow \widehat{\mathbf{R}}$ extends by continuity to a homeomorphism of $\widehat{\mathbf{R}}$ because $\widehat{\mathbf{Q}}, \tau^{0}$ are dense in $\widehat{\mathbf{R}}$ and $h_{\tau}$ is monotone on $\widehat{\mathbf{Q}}$. 
Given a decorated tesselation $\tilde{\tau}$ together with a distinguished oriented geodesic $e \in \tau$, Penner [14] assigns to each geodesic $f \in \mathcal{F}$ a positive number as follows. Let $C_{1}$ and $C_{2}$ be horocycles of the decoration $\tilde{\tau}$ based at the endpoints of $h_{\tau}(f) \in \tau$. Let $\delta(f)$ be a signed hyperbolic distance between $M_{1}=h_{\tau}(f) \cap C_{1}$ and $M_{2}=h_{\tau}(f) \cap C_{2}$, where the sign is positive if the arc of $h_{\tau}(f)$ between $M_{1}$ and $M_{2}$ is outside $C_{1}$ and $C_{2}$, otherwise the sign is negative (see [14]). The lambda length of $f \in \mathcal{F}$ is given by

$$
\lambda(f)=e^{-2 \delta(f)} .
$$

This introduces the lambda length function $\lambda: \mathcal{F} \rightarrow \mathbf{R}^{+}$for any decorated tesselation $\tilde{\tau}$ (see Penner [15]). Let $e, e^{\prime} \in \tau$ be adjacent geodesics. Then $h_{\tau}: \mathcal{F} \rightarrow \tau$ maps adjacent edges $f, f^{\prime} \in \mathcal{F}$ onto $e, e^{\prime}$, respectively. We define horocyclic length $\alpha\left(f, f^{\prime}\right)$ to be the length of the arc of the horocycle from $\tilde{\tau}$ with center the common endpoint of $e$ and $e^{\prime}$ that lies inside the hyperbolic wedge with boundary sides $e, e^{\prime}$.

Given a map $\lambda: \mathcal{F} \rightarrow \mathbf{R}^{+}$there exists a monotone map $h_{\lambda}: \widehat{\mathbf{Q}} \rightarrow \widehat{\mathbf{R}}$, called the characteristic map of $\lambda$, and a decoration (ie choice of horocycles) on $h_{\lambda}(\hat{\mathbf{Q}})$ such that the lambda length of $h_{\lambda}(f)$ with respect to the decoration is equal to $\lambda(f)$. The characteristic map $h_{\lambda}: \hat{\mathbf{Q}} \rightarrow \widehat{\mathbf{R}}$ does not always extend to a homeomorphism similar to the case of shears. It is a fundamental question in this theory to give necessary and sufficient condition on the map $\lambda: \mathcal{F} \rightarrow \mathbf{R}$ such that $h_{\lambda}$ extends by continuity to a homeomorphism or perhaps to a quasisymmetric map. Penner and Sullivan [15, Theorem 6.4] gave a sufficient condition on the lambda lengths to induce a quasisymmetric map as follows. A lambda length function $\lambda: \mathcal{F} \rightarrow \mathbf{R}$ is said to be pinched if there exists $K>1$ such that

$$
\frac{1}{K} \leq \lambda(f) \leq K
$$

for all $f \in \mathcal{F}$. Penner and Sullivan showed that if $\lambda: \mathcal{F} \rightarrow \mathbf{R}$ is pinched then the characteristic map $h_{\lambda}$ extends to a quasisymmetric map of $\widehat{\mathbf{R}}$ [15, Theorem 6.4].

In Theorem E we give a necessary and sufficient condition such that $h_{\lambda}$ is a quasisymmetric (as well as a symmetric) map of $\widehat{\mathbf{R}}$.

Proof of Theorem $\mathbf{E}$ Let $\tau=h_{\lambda}(\mathcal{F})$ be the ideal geodesic triangulation (tesselation) corresponding to the lambda lengths $\lambda$ and let $\tilde{\tau}$ be the decorated tesselation corresponding to $\lambda$ (see Penner [15] for the construction). Let $s: \mathcal{F} \rightarrow \mathbf{R}$ be shear function corresponding to $h_{\lambda}$ and let $\left\{e_{n}\right\}_{n \in \mathbf{Z}}$ be a fan of geodesics in $\mathcal{F}$ with tip $p$. Then we have

$$
s(p ; m, k)=\frac{\alpha\left(e_{m}, e_{m+1}\right)+\alpha\left(e_{m+1}, e_{m+2}\right)+\cdots+\alpha\left(e_{m+k}, e_{m+k+1}\right)}{\alpha\left(e_{m}, e_{m-1}\right)+\alpha\left(e_{m-1}, e_{m-2}\right)+\cdots+\alpha\left(e_{m-k}, e_{m-k-1}\right)} .
$$

Theorem E immediately follows from Theorem A. 
In Theorem D, we find a necessary and sufficient condition such that $h_{\lambda}$ extends to a homeomorphism of $\hat{\mathbf{R}}$. The criteria follows from the proof of Theorem $\mathrm{C}$ and it is obtained by calculating the length of $l\left(P_{1}\right)$ in terms of horocyclic and lambda lengths. Since the horocyclic lengths are expressed in terms of the lambda lengths, the formula can be written only in terms of the lambda lengths although we do not do this.

Proof of Theorem D Let $\lambda: \mathcal{F} \rightarrow \mathbf{R}^{+}$be a lambda length function and let $h_{\lambda}: \hat{\mathbf{Q}} \rightarrow \widehat{\mathbf{R}}$ be the corresponding characteristic map. Denote by $\tau$ the image tesselation $h_{\lambda}(\mathcal{F})$ and by $\tilde{\tau}$ the decoration which realizes the lambda lengths $\lambda$.

Let $\left\{e_{n}\right\}_{n \in \mathbf{N}}$ be an arbitrary chain in $\mathcal{F}$. Denote by $\lambda_{n}=\lambda\left(e_{n}\right)$ the lambda length of $e_{n}$. Then $\lambda_{n}=e^{-2 \delta_{n}}$, where $\delta_{n}$ is the signed hyperbolic distance between the horocycles of $\tilde{\tau}$ with centers at the endpoints of $e_{n}$. Thus $\lambda_{n}^{-1 / 2}=e^{\delta_{n}}$. Let $W_{n}$ be the wedge with boundary sides $h_{\lambda}\left(e_{n}\right)$ and $h_{\lambda}\left(e_{n+1}\right)$ and let $C_{n}$ be the horocycle of the decoration $\tilde{\tau}$ with center at the common endpoint of $h_{\lambda}\left(e_{n}\right)$ and $h_{\lambda}\left(e_{n+1}\right)$. Let $\alpha_{n}$ be the horocyclic length for the wedge with boundaries $e_{n}$ and $e_{n+1}$ namely the length of $C_{n} \cap W_{n}$. Let $l_{n}$ be the length of $l\left(P_{1}\right) \cap W_{n}$, where $P_{1}$ is chosen such that $l_{1}=\lambda_{1}^{-1 / 2} \alpha_{1}=e^{\delta_{1}} \alpha_{1}$.

We need to show that

$$
l_{n}=\left(\lambda_{n}^{-1 / 2} \lambda_{n-1}^{1 / 2} \cdots \lambda_{1}^{(-1)^{n} / 2}\right) \alpha_{n} .
$$

Elementary hyperbolic considerations shows that $l_{n}=e^{d_{n}} \alpha_{n}$ where $d_{n}$ is the signed distance from $l\left(P_{1}\right) \cap W_{n}$ to the horocycle $C_{n}$ such that $d_{n}>0$ if $l\left(P_{1}\right) \cap W_{n}$ is outside $C_{n}$ and $d_{n}<0$ otherwise. Thus it remains to show that

$$
e^{d_{n}}=\lambda_{n}^{-1 / 2} \lambda_{n-1}^{1 / 2} \cdots \lambda_{1}^{(-1)^{n} / 2}
$$

We finish the argument by induction on $n$. By our choice of $P_{1}$, we immediately have

$$
e^{d_{1}}=e^{\delta_{1}}=\lambda_{1}^{-1 / 2} \text {. }
$$

Assume that $n>1$ and that

$$
e^{d_{n}}=\lambda_{n}^{-1 / 2} \lambda_{n-1}^{1 / 2} \cdots \lambda_{1}^{(-1)^{n} / 2}
$$

We calculate $e^{d_{n+1}}$. Since $d_{n}$ is the signed distance from $l\left(P_{1}\right) \cap W_{n}$ to $C_{n}$, it follows that the signed distance of $l\left(P_{1}\right) \cap H_{s}\left(e_{n+1}\right)$ to $C_{n}$ is $d_{n}$. Since $\delta_{n+1}$ is the signed distance between $C_{n}$ and $C_{n+1}$, it follows that $d_{n+1}=\delta_{n+1}-d_{n}$. This gives the desired formula. 


\section{References}

[1] W Abikoff, C J Earle, S Mitra, Barycentric extensions of monotone maps of the circle, from: "In the tradition of Ahlfors and Bers, III", (W Abikoff, A Haas, editors), Contemp. Math. 355, Amer. Math. Soc. (2004) 1-20 MR2145053

[2] L V Ahlfors, Lectures on quasiconformal mappings, Van Nostrand Math. Studies 10, Van Nostrand, Toronto-New York-London (1966) MR0200442 Manuscript prepared with the assistance of C J Earle

[3] L Bers, Universal Teichmüller space, from: "Analytic methods in mathematical physics (Sympos., Indiana Univ., Bloomington, Ind., 1968)", (R P Gilbert, R G Newton, editors), Gordon and Breach, New York (1970) 65-83 MR0349988

[4] F Bonahon, Shearing hyperbolic surfaces, bending pleated surfaces and Thurston's symplectic form, Ann. Fac. Sci. Toulouse Math. (6) 5 (1996) 233-297 MR1413855

[5] A Douady, C J Earle, Conformally natural extension of homeomorphisms of the circle, Acta Math. 157 (1986) 23-48 MR857678

[6] C J Earle, F P Gardiner, N Lakic, Asymptotic Teichmüller space. II. The metric structure, from: "In the tradition of Ahlfors and Bers, III", (W Abikoff, A Haas, editors), Contemp. Math. 355, Amer. Math. Soc. (2004) 187-219 MR2145063

[7] C J Earle, V Markovic, D Saric, Barycentric extension and the Bers embedding for asymptotic Teichmüller space, from: "Complex manifolds and hyperbolic geometry (Guanajuato, 2001)”, (C J Earle, W J Harvey, S Recillas-Pishmish, editors), Contemp. Math. 311, Amer. Math. Soc. (2002) 87-105 MR1940165

[8] F P Gardiner, Y Jiang, Asymptotically affine and asymptotically conformal circle endomorphisms, from: "Infinite dimensional Teichmüller spaces and moduli spaces", (E Fujikawa, editor), RIMS Kôkyûroku Bessatsu B17, RIMS, Kyoto (2010) 37-54

[9] F P Gardiner, N Lakic, Quasiconformal Teichmüller theory, Math. Surveys and Monogr. 76, Amer. Math. Soc. (2000) MR1730906

[10] F P Gardiner, D P Sullivan, Symmetric structures on a closed curve, Amer. J. Math. 114 (1992) 683-736 MR1175689

[11] É Ghys, Groups acting on the circle, Enseign. Math. (2) 47 (2001) 329-407 MR1876932

[12] J Kahn, V Markovic, Random ideal triangulations and the Weil-Petersson distance between finite degree covers of punctured Riemann surfaces arXiv:0806.2304

[13] V Markovic, Quasisymmetric groups, J. Amer. Math. Soc. 19 (2006) 673-715 MR2220103

[14] R C Penner, The decorated Teichmüller space of punctured surfaces, Comm. Math. Phys. 113 (1987) 299-339 MR919235 
[15] R C Penner, Universal constructions in Teichmüller theory, Adv. Math. 98 (1993) 143-215 MR1213724

[16] WP Thurston, Minimal stretch maps between hyperbolic surfaces arXiv: math.GT/9801039

[17] W P Thurston, Three-dimensional geometry and topology. Vol. 1, (S Levy, editor), Princeton Math. Series 35, Princeton Univ. Press (1997) MR1435975

Department of Mathematics, Queens College, CUNY

65-30 Kissena Blvd, Kiely Hall, Room 237, Flushing NY 11367, USA

saric@math . sunysb . edu

http://qcpages.qc. cuny.edu/ dsaric/

Proposed: Jean-Pierre Otal

Received: 11 October 2009

Seconded: Benson Farb, Walter Neumann Accepted: 6 September 2010 